\title{
Resampling-Based Analysis of Multivariate Data and Repeated Measures Designs with the R Package MANOVA.RM
}

\author{
by Sarah Friedrich, Frank Konietschke and Markus Pauly
}

\begin{abstract}
Nonparametric statistical inference methods for a modern and robust analysis of longitudinal and multivariate data in factorial experiments are essential for research. While existing approaches that rely on specific distributional assumptions of the data (multivariate normality and/or equal covariance matrices) are implemented in statistical software packages, there is a need for user-friendly software that can be used for the analysis of data that do not fulfill the aforementioned assumptions and provide accurate $p$ value and confidence interval estimates. Therefore, newly developed nonparametric statistical methods based on bootstrap- and permutation-approaches, which neither assume multivariate normality nor specific covariance matrices, have been implemented in the freely available $\mathrm{R}$ package MANOVA.RM. The package is equipped with a graphical user interface for plausible applications in academia and other educational purpose. Several motivating examples illustrate the application of the methods.
\end{abstract}

\section{Introduction}

Nowadays, a large amount of measurements are taken per experimental unit or subject in many experimental studies-requiring inferential methods from multivariate analysis in a unified way. Here we distinguish between two cases:

1. If the same quantity is measured under different treatment conditions or at different time points, a repeated measures (RM) design is present. Therein, observations are measured on the same scale and are combinable. This is also the case if the measuring instrument produces multiple responses, e.g., microarrays in bioinformatics.

2. If different quantities are measured on the same unit or subject, a multivariate analysis of variance (MANOVA) design is apparent. In such a situation, data is measured on different scales and not combinable (e.g., height and weight).

These two different definitions do not only lead to different questions of interest but also require different inference procedures as outlined below. In particular, the main difference between the two approaches is that in repeated measures designs comparisons between the response variables are meaningful. This means that also hypotheses regarding sub-plot or within subject factors (e.g., time) are of interest. On the other hand, MANOVA settings are usually designed to detect effects of the observed factors (and interactions thereof) on the multivariate outcome vectors, thus allowing -in contrast to multiple univariate ANOVA analyses-to evaluate the combined changes of the outcome variables with respect to the factor levels.

Despite their differences, MANOVA- and RM-type techniques share the same advantages over classical univariate endpoint-wise-ANOVA-type-analyses:

- They provide joint inference and take the dependency across the endpoints into account, thus leading to possibly larger power to detect underlying effects.

- They allow for testing of additional factorial structures and

- can easily be equipped with a closed testing procedure for subsequently detecting local effects in specific components, i.e. to perform post-hoc analyses.

Focusing on metric data and mean-based procedures, MANOVA and RM models are typically inferred by means of "classical" procedures such as Wilks' Lambda, Lawley-Hotelling, Roy's largest root (Davis, 2002; Johnson and Wichern, 2007; Anderson, 2001) or (generalized) linear mixed models with generalized estimating equations. For the classical one-way layout, these methods are implemented in $\mathrm{R}$ within the manova function in the stats package, where one can choose between the options 'Pillai', 'Wilks', 'Hotelling-Lawley' and 'Roy'. Nonparametric rank-based methods for null hypotheses formulated in distribution functions are implemented within the packages npmv for oneand two-way MANOVA (Burchett et al., 2017) and nparLD for several repeated measures designs 
(Noguchi et al., 2012). In case of fixed block effects, the GFD package (Friedrich et al., 2017b), which implements a permutation Wald-type test in the univariate setting, can also be used.

(Generalized) linear mixed models are implemented in the $1 \mathrm{~m}$ and the glm function (package stats) for univariate data as well as in the SCGLR package for Generalized Linear Model estimation in the context of multivariate data (Cornu et al., 2018). The Anova and Manova function in the car package (Fox and Weisberg, 2011) calculate type-II and type-III analysis-of-variance tables for objects produced by, e.g., $1 \mathrm{~m}$, glm or manova in the univariate and multivariate context, respectively. In the MANOVA context, repeated measures designs can be included as well.

Furthermore, the packages flip (Finos et al., 2018) and ffmanova (Øyvind Langsrud and Mevik, 2019) contain interesting permutation and rotation tests, which, however, require certain invariances resulting in model restrictions (see, e.g., the discussion in Huang et al., 2006; Chung and Romano, 2013) .

Most of these procedures, however, rely on specific distributional assumptions (such as multivariate normality) and/or specific covariance or correlation structures (e.g., homogeneity between groups or, for RM, compound symmetry; possibly implying equal correlation between measurements) which may often not be justifiable in real data. In particular, with decreasing sample sizes and increasing dimensions, such presumptions are almost impossible to verify in practice and may lead to inflated type-I-errors, cf. Vallejo et al. (2001); Lix and Keselman (2004); Vallejo Seco et al. (2007); Livacic-Rojas et al. (2010). To this end, several alternative procedures have been developed that tackle the above problems and have been compared in extensive simulation studies, see amongst others Brunner (2001); Lix and Lloyd (2007); Gupta et al. (2008); Zhang (2011); Harrar and Bathke (2012); Konietschke et al. (2015); Xiao and Zhang (2016); Bathke et al. (2018); McFarquhar et al. (2016); Friedrich et al. (2017a); Livacic-Rojas et al. (2017); Friedrich and Pauly (2018) and the references cited therein. Here, we focus on nonparametric statistical methods that are valid in the multivariate Behrens-Fisher situation-equal covariance matrices across the groups are not assumed-and provide accurate inferential results in terms of $p$ value estimates and confidence intervals for the parameters of interest. In particular, we implemented bootstrap- and permutation-based approaches to approximate the distribution of the test statistics in a robust way. Simulation studies comparing these approaches to the traditional methods mentioned above can, e. g. be found in the main papers and the supplements of Friedrich et al. (2017a) and Bathke et al. (2018).

More precisely, we focus on nonparametric methods for testing main and interaction effects of fixed factors in repeated measures designs and multivariate data. In particular, general Wald-type test statistics (for MANOVA and RM), ANOVA-type statistics (for RM) and modified ANOVA-type tests (for MANOVA) are implemented in MANOVA.RM (Friedrich et al., 2019) because

- they can be used to test hypotheses in various factorial designs in a flexible way,

- their sampling distribution can be approximated by resampling techniques, even allowing their application for small sample sizes,

- and they are appropriate methods in the Behrens-Fisher situation.

To make the methods freely accessible we have provided the R package MANOVA.RM for routine statistical analyses. It is available from the R Archive at

https://CRAN.R-project.org/package=MANOVA.RM

The main functions RM (for RM designs) and MANOVA (for MANOVA designs) are developed in style of the well known ANOVA functions Im or aov. Its user-friendly application not only provides the $p$ values and test statistics of interest but also a descriptive overview together with component-wise two-sided confidence intervals. Moreover, the MANOVA function even allows for an easy calculation and confidence ellipsoid plots for specified multivariate contrasts as described in Friedrich and Pauly (2018).

Specifically, for testing multivariate main- and interaction effects in one-, two- and higher-way MANOVA models, the MANOVA function provides

- the Wald-type statistic (WTS) proposed by Konietschke et al. (2015) using a parametric bootstrap, a wild bootstrap or its asymptotic $\chi^{2}$-distribution for $p$ value computations, and

- the modified ANOVA-type statistic (MATS) proposed by Friedrich and Pauly (2018) using a parametric or wild bootstrap procedure for $p$ value computations.

In addition to multivariate group-wise effects, the RM function also allows to test hypotheses formulated across within subject factors. The implemented test statistics are

- the ANOVA-type statistic (ATS) using an F-approximation as considered in Brunner (2001) as well as a parametric and a wild bootstrap approach and 
- the Wald-type statistic (WTS) using the asymptotic $\chi^{2}$-distribution (Brunner, 2001), the permutation technique proposed in Friedrich et al. (2017a) as well as a parametric (Bathke et al., 2018) and a wild bootstrap approach for $p$ value estimation.

The paper is organized as follows: In Section 2.2 the multivariate statistical model as well as the implemented inference procedures are described. The application of the R package MANOVA.RM is exemplified on several Repeated Measures and MANOVA Examples in Section 2.3. Finally, the paper closes with a discussion in Section 2.4.

Throughout the paper we use the subsequent notation from multivariate linear models: For $a \in \mathbb{N}$ we denote by $\boldsymbol{P}_{a}=\boldsymbol{I}_{a}-\frac{1}{a} \boldsymbol{J}_{a}$ the $a$-dimensional centering matrix, by $\boldsymbol{I}_{a}$ the $a$-dimensional identity matrix and by $\boldsymbol{J}_{a}$ the $a \times a$ matrix of $1^{\prime}$ 's, i.e., $\boldsymbol{J}_{a}=\mathbf{1}_{a} \mathbf{1}_{a}^{\prime}$, where $\mathbf{1}_{a}=(1, \ldots, 1)^{\prime}$ is the $a$-dimensional column vector of 1 's.

\section{Statistical model and inference methods}

For both the RM and the MANOVA design equipped with an arbitrary number of fixed factors, we consider the general linear model given by $d$-variate random vectors

$$
\boldsymbol{X}_{i k}=\left(X_{i j k}\right)_{j=1}^{d}=\boldsymbol{\mu}_{i}+\boldsymbol{\epsilon}_{i k}
$$

Here, $k=1, \ldots, n_{i}$ denotes the experimental unit or subject in group $i=1, \ldots, a$. Note, that a higher-way factorial structure on the groups/between subject or within subject factors can be achieved by sub-indexing the indices $i$ (group/between subject factors) or $j$ (within subject factors) into $i_{1}, \ldots, i_{p}$ or $j_{1}, \ldots, j_{q}$. In this model $\mu_{i}=\left(\mu_{i 1}, \ldots, \mu_{i d}\right)^{\prime} \in R^{d}$ is the mean vector in group $i=1, \ldots, a$ and for each fixed $i$ it is assumed that the error terms $\epsilon_{i k}, k=1, \ldots, n_{i}$, are independent and identically distributed $d$-variate random vectors with mean $E\left(\epsilon_{i 1}\right)=0$ and existing variances $0<\sigma_{i j}^{2}=\operatorname{var}\left(X_{i j k}\right)<\infty, j=1, \ldots, d$. For the WTS-type procedures we additionally assume positive definite covariance matrices $\operatorname{cov}\left(\boldsymbol{\epsilon}_{i 1}\right)=\boldsymbol{V}_{i}>0$ and existing finite fourth moments $E\left(\left\|\boldsymbol{\epsilon}_{i 1}\right\|^{4}\right)<\infty$.

Within this framework, hypotheses for RM or MANOVA can be formulated by means of an adequate contrast hypothesis matrix $\boldsymbol{H}$ by

$$
H_{0}: H \boldsymbol{\mu}=\mathbf{0}
$$

where $\mu=\left(\mu_{1}, \ldots, \mu_{a}\right)^{\prime}$.

Let $\overline{\boldsymbol{X}}_{\bullet}=\left(\overline{\boldsymbol{X}}_{1 .}^{\prime}, \ldots, \overline{\boldsymbol{X}}_{a}^{\prime}\right)^{\prime}$ denote the vector of pooled group means $\overline{\boldsymbol{X}}_{i .}=\frac{1}{n_{i}} \sum_{k=1}^{n_{i}} \boldsymbol{X}_{i k}, i=1, \ldots, a$ and $\widehat{\boldsymbol{\Sigma}}_{N}=N \cdot \operatorname{diag}\left\{\widehat{\boldsymbol{V}}_{1} / n_{1}, \ldots, \widehat{\boldsymbol{V}}_{a} / n_{a}\right\}$ the estimated covariance of $\sqrt{N} \overline{\boldsymbol{X}}_{\bullet}$. Here, $N=\sum_{i} n_{i}$ and $\widehat{\boldsymbol{V}}_{i}=\frac{1}{n_{i}-1} \sum_{k=1}^{n_{i}}\left(\boldsymbol{X}_{i k}-\overline{\boldsymbol{X}}_{i}\right.$. $\left(\boldsymbol{X}_{i k}-\overline{\boldsymbol{X}}_{i}\right)^{\prime}$. In this set-up Konietschke et al. (2015) propose a statistic of Wald-type (WTS)

$$
T_{N}=T_{N}(\boldsymbol{X})=N \overline{\boldsymbol{X}}_{\bullet}^{\prime} \boldsymbol{T}\left(\boldsymbol{T} \widehat{\boldsymbol{\Sigma}}_{N} \boldsymbol{T}\right)^{+} \boldsymbol{T} \overline{\boldsymbol{X}}_{\bullet},
$$

for testing $H_{0}$, where $\boldsymbol{T}=\boldsymbol{H}^{\prime}\left(\boldsymbol{H H}^{\prime}\right)^{+} \boldsymbol{H}, \boldsymbol{X}=\left\{\boldsymbol{X}_{11}, \ldots, \boldsymbol{X}_{a n_{a}}\right\}$, and $\boldsymbol{A}^{+}$denotes the Moore-Penrose inverse of the matrix $\boldsymbol{A}$. Since its asymptotic $\chi_{\operatorname{rank}(\boldsymbol{T})}^{2}$ null distribution provides a poor finite sample approximation, they propose the following asymptotic model-based bootstrap approach: Given the data $\boldsymbol{X}$ let $\boldsymbol{X}_{i k}^{\star} \sim N\left(\mathbf{0}, \widehat{\boldsymbol{V}}_{i}\right), i=1, \ldots, a, k=1, \ldots, n_{i}$, be independent random vectors that are used for recalculating the test statistic as $T_{N}^{\star}=T_{N}\left(\boldsymbol{X}^{\star}\right)$, where $\boldsymbol{X}^{\star}=\left\{\boldsymbol{X}_{11}^{\star}, \ldots, \boldsymbol{X}_{a n_{a}}^{\star}\right\}$. Denoting by $c^{\star}$ the corresponding $(1-\alpha)$-quantile of the (conditional) distribution of $T_{N}^{\star}$ the test rejects $H_{0}$ if $T_{N}>c^{\star}$. The validity of this procedure (also named parametric bootstrap WTS) is proven in Konietschke et al. (2015).

This procedure is not only applicable for MANOVA but also for RM designs (Bathke et al., 2018). However, Friedrich et al. (2017a) proposed a more favourable technique for Repeated Measurements. It is based on an at first blush chaotic resampling method: Wild permutation of all pooled components without taking group membership or possible dependencies into account. Denoting the resulting permuted data set as $\boldsymbol{X}^{\pi}$ their permutation test for RM models rejects $H_{0}$ if $T_{N}>c^{\pi}$. Here $c^{\pi}$ denotes the $(1-\alpha)$-quantile of the (conditional) distribution of the permutation version of the test statistic $T_{N}^{\pi}=T_{N}\left(\boldsymbol{X}^{\pi}\right)$. As shown in extensive simulations in Friedrich et al. (2017a) and the corresponding supporting information this 'wild' permutation WTS method controls the type-I error rate very well. Note that this procedure is only applicable for RM due to the commensurate nature of their components. In MANOVA set-ups the permutation would stir different scalings making comparisons meaningless.

In addition to these WTS procedures two other statistics are considered as well. For RM the 
well-established ANOVA-type statistic (ATS)

$$
Q_{N}=N \bar{X}_{\bullet}^{\prime} T \bar{X}
$$

by Brunner (2001) is implemented together with the enhanced F-approximation of the statistic proposed in Brunner et al. $(1997,2002)$ and implemented in the SAS PROC Mixed procedure. Although known to be rather conservative it has the advantage (over the WTS) of being applicable in case of eventually singular covariance matrices $V_{i}$ or $\widehat{V}_{i}$ since it waives the Moore-Penrose inverse involved in Equation 2.

Similar to the permuted WTS the ATS given in Equation 3 is only applicable for RM since it is not invariant under scale transformations (e.g., change of units) of the univariate components. To nevertheless provide a robust method for MANOVA settings which is also applicable in case of singular $\boldsymbol{V}_{i}$ or $\widehat{\boldsymbol{V}}_{i}$, Friedrich and Pauly (2018) have recently proposed the novel MATS (modified ATS)

$$
M_{N}=M_{N}(\boldsymbol{X})=N \bar{X}_{\bullet}^{\prime} T\left(T \widehat{D}_{N} \boldsymbol{T}\right)^{+} \boldsymbol{T} \bar{X} \bullet
$$

Here, the involved diagonal matrix $\widehat{\boldsymbol{D}}_{N}=\oplus_{1 \leq i \leq a, 1 \leq s \leq d} N \widehat{\sigma}_{i s}^{2} / n_{i}$ of the empirical variances $\widehat{\sigma}_{i s}^{2}$ of component $s$ in group $i$, deduces an invariance under component-wise scale transformations of the MATS for null hypotheses as described in Section 2.2.1, i.e., of the form $\boldsymbol{T}=\boldsymbol{M} \otimes \boldsymbol{I}_{d}$, see Friedrich and Pauly (2018) for details. To obtain an accurate finite sample performance, it is also equipped with an asymptotic model based bootstrap approach. That is, MATS rejects $H_{0}$ if $M_{N}>\tilde{c}^{\star}$, where $\tilde{c}^{\star}$ is the $(1-$ $\alpha$ )-quantile of the (conditional) distribution of the bootstrapped statistic $M_{N}^{\star}=M_{N}\left(X^{\star}\right)$. In addition, we implemented a wild bootstrap approach, which is based on multiplying the centered data vectors $\left(\boldsymbol{X}_{i k}-\boldsymbol{X}_{i}\right.$.) with random weights $W_{i k}$ fulfilling $E\left(W_{i k}\right)=0, \operatorname{var}\left(W_{i k}\right)=1$ and $\sup _{i, k} E\left(W_{i k}^{4}\right)<\infty$. In the package, we implemented Rademacher distributed weights, i.e., random signs. Extensive simulations in Friedrich and Pauly (2018) not only confirm its applicability in case of singular covariance matrices but also disclose a very robust behaviour that even seems to be advantageous over the parametrically bootstrapped WTS of Konietschke et al. (2015). However, both procedures, as well as the 'usual' asymptotic WTS are displayed within the MANOVA functions. All of the aforementioned procedures are applicable in various factorial designs in a unified way, i.e., when more than one factor may impact the response. The specific models and the hypotheses being tested will be discussed in the next section.

\section{Special designs and hypotheses}

In order to provide a general overview of different statistical designs and layouts that can be analyzed with MANOVA.RM we exemplify few designs that occur frequently in practical applications and discuss the model, hypotheses and limitations. All of the methods implemented in MANOVA.RM are even applicable in higher-way layouts than being presented here; and the list should not be seen as the limited application of the package. The models are derived by sub-indexing the index $i$ in Equation 1 in the following ways:

- One-Way (A): Writing $\mu_{i}=\boldsymbol{v}+\boldsymbol{\alpha}_{i}$ we have $X_{i k}=\boldsymbol{v}+\boldsymbol{\alpha}_{i}+\boldsymbol{\epsilon}_{i k}$ with $\sum_{i=1}^{a} \boldsymbol{\alpha}_{i}=\mathbf{0}$ and obtain the null hypothesis of 'no group' or 'factor $A^{\prime}$ effect as

$$
\begin{aligned}
H_{0}(\boldsymbol{A}):\left\{\left(\boldsymbol{P}_{a} \otimes \boldsymbol{I}_{d}\right) \boldsymbol{\mu}=\mathbf{0}\right\} & =\left\{\boldsymbol{\mu}_{1}=\cdots=\boldsymbol{\mu}_{a}\right\} \\
& =\left\{\boldsymbol{\alpha}_{1}=\cdots=\boldsymbol{\alpha}_{a}=\mathbf{0}\right\} .
\end{aligned}
$$

In case of $a=2$ this includes the famous multivariate Behrens-Fisher problem as, e.g., analyzed in Yao (1965); Nel and Van der Merwe (1986); Christensen and Rencher (1997); Krishnamoorthy and Yu (2004) or Yanagihara and Yuan (2005).

- Crossed Two-Way $(\boldsymbol{A} \times \boldsymbol{B})$ : Splitting the index into two and writing $\boldsymbol{\mu}_{i j}=\boldsymbol{v}+\boldsymbol{\alpha}_{i}+\boldsymbol{\beta}_{j}+\gamma_{i j}$ we obtain the model $\boldsymbol{X}_{i j k}=\boldsymbol{v}+\boldsymbol{\alpha}_{i}+\boldsymbol{\beta}_{j}+\gamma_{i j}+\boldsymbol{\epsilon}_{i j k}, 1 \leq i \leq a, 1 \leq j \leq b, 1 \leq k \leq n_{i j}$ with $\sum_{i} \boldsymbol{\alpha}_{i}=\sum_{j} \boldsymbol{\beta}_{j}=\sum_{i} \gamma_{i j}=\sum_{j} \gamma_{i j}=\mathbf{0}$. The corresponding null hypotheses of no main effects in $\boldsymbol{A}$ or $\boldsymbol{B}$ and no interaction effect between $\boldsymbol{A}$ and $\boldsymbol{B}$ can be written as:

$$
\begin{array}{cl}
H_{0}(\boldsymbol{A}):\left\{\left(\boldsymbol{P}_{a} \otimes b^{-1} \boldsymbol{J}_{b} \otimes \boldsymbol{I}_{d}\right) \boldsymbol{\mu}=\mathbf{0}\right\} & =\left\{\boldsymbol{\alpha}_{1}=\cdots=\boldsymbol{\alpha}_{a}=\mathbf{0}\right\}, \\
H_{0}(\boldsymbol{B}):\left\{\left(a^{-1} \boldsymbol{J}_{a} \otimes \boldsymbol{P}_{b} \otimes \boldsymbol{I}_{d}\right) \boldsymbol{\mu}=\mathbf{0}\right\} & =\left\{\boldsymbol{\beta}_{1}=\cdots=\boldsymbol{\beta}_{b}=\mathbf{0}\right\}, \\
H_{0}(\boldsymbol{A B}):\left\{\left(\boldsymbol{P}_{a} \otimes \boldsymbol{P}_{b} \otimes \boldsymbol{I}_{d}\right) \boldsymbol{\mu}=\mathbf{0}\right\} & =\left\{\gamma_{11}=\cdots=\gamma_{a b}=\mathbf{0}\right\} .
\end{array}
$$

Note that the interpretation of main effects is complicated by the presence of significant interaction effects and further analyses are necessary to determine the direction of the effects.

- Hierarchically nested Two-Way $(B(A))$ : A fixed subcategory $B$ within factor $A$ can be introduced via the model $\boldsymbol{X}_{i j k}=\boldsymbol{v}+\boldsymbol{\alpha}_{i}+\boldsymbol{\beta}_{j(i)}+\boldsymbol{\epsilon}_{i j k}, 1 \leq i \leq a, 1 \leq j \leq b_{i}, 1 \leq k \leq n_{i j}$ with 
$\sum_{i} \boldsymbol{\alpha}_{i}=\sum_{j} \boldsymbol{\beta}_{j(i)}=\mathbf{0}$. Here, the hypotheses of no main effect $A$ or no sub-category main effect $B$ can be written as

$$
\begin{aligned}
H_{0}(A):\left\{\left(\boldsymbol{P}_{a} \tilde{\boldsymbol{J}}_{b} \otimes \boldsymbol{I}_{d}\right) \boldsymbol{\mu}=\mathbf{0}\right\} & =\left\{\boldsymbol{\alpha}_{1}=\cdots=\boldsymbol{\alpha}_{a}=\mathbf{0}\right\} \\
H_{0}(B(A)):\left\{\left(\widetilde{\boldsymbol{P}}_{b} \otimes \boldsymbol{I}_{d}\right) \boldsymbol{\mu}=\mathbf{0}\right\} & =\left\{\boldsymbol{\beta}_{j(i)}=0 \forall 1 \leq i \leq a, 1 \leq j \leq b_{i}\right\}
\end{aligned}
$$

with $\widetilde{\boldsymbol{P}}_{b}=\bigoplus_{j=1}^{a} \boldsymbol{P}_{b_{j}}, \widetilde{\boldsymbol{J}}_{b}=\bigoplus_{j=1}^{a} b_{j}^{-1} \mathbf{1}_{b_{j}}^{\prime}$ and $\boldsymbol{\mu}:=\left(\boldsymbol{\mu}_{11}^{\prime}, \ldots, \boldsymbol{\mu}_{1 b_{1},}^{\prime}, \boldsymbol{\mu}_{21}^{\prime}, \ldots, \boldsymbol{\mu}_{2 b_{2}}^{\prime}, \ldots, \boldsymbol{\mu}_{a b_{a}}^{\prime}\right)^{\prime}$.

We only implemented balanced designs, i.e., $b_{i}=b$ for all $i=1, \ldots, a$. Hierarchically nested three-way designs or arbitrary crossed higher-way layouts can be introduced similarly and are implemented as well.

- Repeated Measures and Split Plot Designs are covered by setting $d=t$, where even hypotheses about within subject factors can be formulated. We exemplify this for profile analyses in the special case of a one-sample RM design with $a=1$

$$
H_{0} \text { (Time) }:\left\{\boldsymbol{P}_{t} \boldsymbol{\mu}=\mathbf{0}\right\}=\left\{\mu_{11}=\cdots=\mu_{1 t}\right\},
$$

as well as for a two-sample RM design with $a=2$ :

$$
\begin{aligned}
H_{0}(\text { Parallel }):\left\{\boldsymbol{T}_{P} \boldsymbol{\mu}=\mathbf{0}\right\} & =\left\{\boldsymbol{\mu}_{1}-\boldsymbol{\mu}_{2}=\gamma \mathbf{1}_{t} \text { for some } \gamma \in \mathbb{R}\right\} \\
H_{0} \text { (Flat) }:\left\{\boldsymbol{T}_{F} \boldsymbol{\mu}=\mathbf{0}\right\} & =\left\{\boldsymbol{\mu}_{1 s}+\boldsymbol{\mu}_{2 s}=\overline{\boldsymbol{\mu}}_{1 .}+\overline{\boldsymbol{\mu}}_{2 .} \text { for all } s\right\} \\
H_{0}(\text { Identical }):\left\{\boldsymbol{T}_{I} \boldsymbol{\mu}=\mathbf{0}\right\} & =\left\{\boldsymbol{\mu}_{1}=\boldsymbol{\mu}_{2}\right\}
\end{aligned}
$$

with $\boldsymbol{T}_{F}=\boldsymbol{P}_{t}\left(\begin{array}{ll}\boldsymbol{I}_{t} & \boldsymbol{I}_{t}\end{array}\right), \boldsymbol{T}_{P}=\left(\begin{array}{ll}\mathbf{1}_{t-1} & -\boldsymbol{I}_{t-1}\end{array}\right) \boldsymbol{T}_{I}$ and $\boldsymbol{T}_{I}=\left(\begin{array}{ll}\boldsymbol{I}_{t} & -\boldsymbol{I}_{t}\end{array}\right)$.

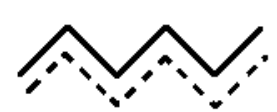

parallel

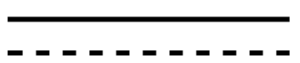

flat identical

Note, that we could also employ more complex factorial structures on the repeated measurements (i.e., more within subject factors) by splitting up the index $j$.

\section{Examples}

To demonstrate the use of the RM and the MANOVA function, we provide several examples for both repeated measures designs and multivariate data in the following. Furthermore, the MANOVA.RM package is equipped with an optional GUI (graphical user interface), based on RGtk2 (Lawrence and Temple Lang, 2010), which will be explained in detail in Section 2.3.3 below.

Both functions are structured similarly: The main input parameters are the formula specifying the outcome on the left hand side and the factor variables of interest on the right, the data and the resampling approach. The latter varies according to the design: the user can choose between a parametric and a wild bootstrap and in the RM design, additionally a permutation approach for the WTS is implemented.

\section{Repeated Measures Designs}

The function RM is built as follows:

R> RM(formula, data, subject, no. subf $=1$, iter $=10000$, alpha $=0.05$,

$+\quad$ resampling $=$ "Perm", $\mathrm{CPU}$, seed, $\mathrm{CI}$.method $=$ "t-quantile", dec $=3$ )

Data need to be provided in long format, i.e., one row per measurement. Here, subject specifies the column name of the subjects variable in the data frame, while no. subf denotes the number of within subject factors considered. Note that in a setting with more than one between subjects factor, the subject ids in the different groups need to be different. Otherwise the program will erroneously assume that these measurements belong to the same subject. The number of cores used for parallel computing as well as a random seed can optionally be specified using CPU and seed, respectively. For calculating the confidence intervals, the user can choose between $t$-quantiles (the default) and the quantiles based on the resampled WTS. The results are rounded to dec digits.

The function RM returns an object of class "RM" from which the user may obtain plots and summaries of the results using $\operatorname{plot}(), \operatorname{print}()$ and summary(), respectively. Here, print() returns a short 
summary of the results, i.e., the values of the test statistics along with degrees of freedom and corresponding $p$ values whereas summary() also displays some descriptive statistics such as the means, sample sizes and confidence intervals for the different factor level combinations. Plotting is based on plotrix (Lemon, 2006). For two- and higher-way layouts, the factors for plotting can be additionally specified in the plot call, see the examples below.

\section{Example 1: One between subject and two within subject factors}

For illustration purposes, we consider the data set o2cons, which is included in MANOVA.RM. This data set contains measurements of the oxygen consumption of leukocytes in the presence and absence of inactivated staphylococci at three consecutive time points. Due to the study design, both time and staphylococci are within subject factors while the treatment (Verum vs. Placebo) is a between subject factor (see Friedrich et al., 2017a, for more details).

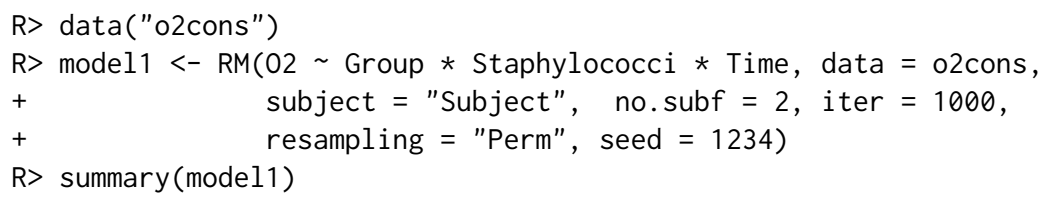




$\begin{array}{ll}\text { Time } & "<0.001 " \\ \text { Group:Time } & "<0.001 " \\ \text { Staphylococci:Time } & " 0.144 " \\ \text { Group:Staphylococci:Time } & " 0.139 "\end{array}$

The output consists of four parts: model1 \$Descriptive gives an overview of the descriptive statistics: The number of observations, mean and confidence intervals are displayed for each factor level combination. Second, model1 \$WTS contains the results for the Wald-type test: The test statistic, degree of freedom and $p$ values based on the asymptotic $\chi^{2}$-distribution are displayed. Note that the $\chi^{2}$-approximation is highly anti-conservative for small sample sizes, cf. Konietschke et al. (2015); Friedrich et al. (2017a). The corresponding results based on the ATS are contained within model1 \$ATS. This test statistic tends to rather conservative decisions in case of small sample sizes and is even asymptotically only an approximation, thus not providing an asymptotic level $\alpha$ test, see Brunner (2001); Friedrich et al. (2017a). Finally, model1 \$resampling contains the $p$ values based on the chosen resampling approach. For the ATS, the permutation approach is not feasible since it would result in an incorrect covariance structure, and is therefore not implemented. Due to the above mentioned issues for small sample sizes, the respective resampling procedure is recommended for such situations.

In this example, we find significant effects of all factors as well as a significant interaction between group and time.

\section{Example 2: Two within subject and two between subject factors}

We consider the data set EEG from the MANOVA.RM package: At the Department of Neurology, University Clinic of Salzburg, 160 patients were diagnosed with either Alzheimer's Disease (AD), mild cognitive impairment (MCI), or subjective cognitive complaints without clinically significant deficits (SCC), based on neuropsychological diagnostics (Bathke et al., 2018). This data set contains $z$-scores for brain rate and Hjorth complexity, each measured at frontal, temporal and central electrode positions and averaged across hemispheres. In addition to standardization, complexity values were multiplied by -1 in order to make them more easily comparable to brain rate values: For brain rate we know that the values decrease with age and pathology, while Hjorth complexity values are known to increase with age and pathology. The three between subject factors considered were sex (men vs. women), diagnosis (AD vs. MCI vs. SCC), and age ( $<70$ vs. $\geq 70$ years). Additionally, the within subject factors region (frontal, temporal, central) and feature (brain rate, complexity) structure the response vector.

Note that due to the small number of subjects in some groups (e.g., only 2 male patients aged $<70$ were diagnosed with $\mathrm{AD}$ ) we restrict our analyses to two between subject factors at a time. However, more complex factorial designs can also be analyzed with MANOVA.RM as outlined above.

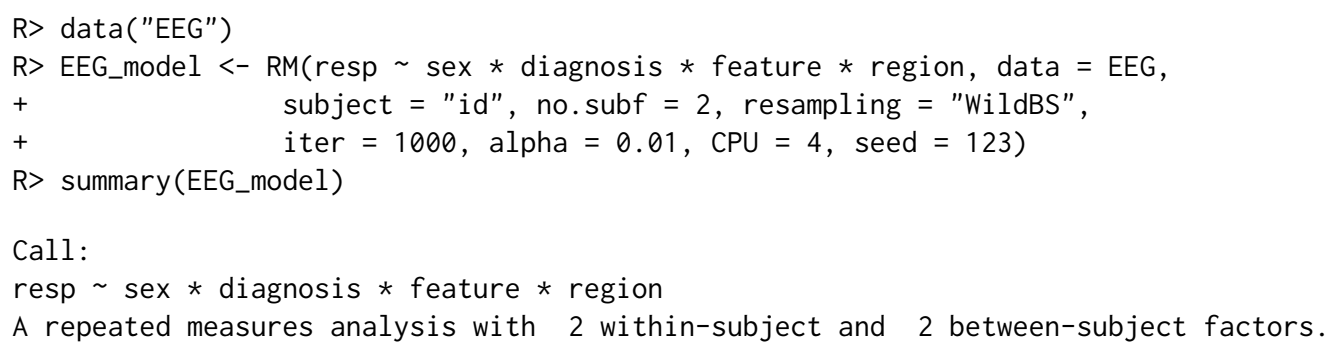

\begin{tabular}{|c|c|c|c|c|c|c|c|c|}
\hline $\operatorname{sex}$ & diagnos & & feature & region & $\mathrm{n}$ & Means & Lower $99 \%$ CI & Upper $99 \%$ CI \\
\hline 1 & M & $A D$ & brainrate & central & 12 & -1.010 & -4.881 & 2.861 \\
\hline 13 & M & $A D$ & brainrate & frontal & 12 & -1.007 & -4.991 & 2.977 \\
\hline 25 & M & $A D$ & brainrate & temporal & 12 & -0.987 & -4.493 & 2.519 \\
\hline 7 & M & $A D$ & complexity & central & 12 & -1.488 & -10.053 & 7.077 \\
\hline 19 & M & $A D$ & complexity & frontal & 12 & -1.086 & -6.906 & 4.735 \\
\hline 31 & M & $A D$ & complexity & temporal & 12 & -1.320 & -7.203 & 4.562 \\
\hline 3 & M & MCI & brainrate & central & 27 & -0.447 & -1.591 & 0.696 \\
\hline 15 & M & MCI & brainrate & frontal & 27 & -0.464 & -1.646 & 0.719 \\
\hline 27 & M & MCI & brainrate & temporal & 27 & -0.506 & -1.584 & 0.572 \\
\hline 9 & M & MCI & complexity & central & 27 & -0.257 & -1.139 & 0.625 \\
\hline 21 & M & MCI & complexity & frontal & 27 & -0.459 & -1.997 & 1.079 \\
\hline 33 & M & MCI & complexity & temporal & 27 & -0.490 & -1.796 & 0.816 \\
\hline 5 & M & SCC & brainrate & central & 20 & 0.459 & -0.414 & 1.332 \\
\hline 17 & M & SCC & brainrate & frontal & 20 & 0.243 & -0.670 & 1.156 \\
\hline
\end{tabular}




$\begin{array}{llrrrrr}29 & \text { M } & \text { SCC brainrate temporal 20 } & 0.409 & -1.210 & 2.028 \\ 11 & \text { M } & \text { SCC complexity central 20 } & 0.349 & -0.070 & 0.767 \\ 23 & \text { M } & \text { SCC complexity frontal 20 } & 0.095 & -1.037 & 1.227 \\ 35 & \text { M } & \text { SCC complexity temporal 20 } & 0.314 & -0.598 & 1.226 \\ 2 & \text { W } & \text { AD brainrate central 24 } & -0.294 & -1.978 & 1.391 \\ 14 & \text { W } & \text { AD brainrate frontal 24 } & -0.159 & -1.813 & 1.495 \\ 26 & \text { W } & \text { AD brainrate temporal 24 } & -0.285 & -1.776 & 1.206 \\ 8 & \text { W } & \text { AD complexity central 24 } & -0.128 & -1.372 & 1.116 \\ 20 & \text { W } & \text { AD complexity frontal 24 } & 0.026 & -1.212 & 1.264 \\ 32 & \text { W } & \text { AD complexity temporal 24 } & -0.194 & -1.670 & 1.283 \\ 4 & \text { W } & \text { MCI brainrate central 30 } & -0.106 & -1.076 & 0.863 \\ 16 & \text { W } & \text { MCI brainrate frontal 30 } & -0.074 & -1.032 & 0.885 \\ 28 & \text { W } & \text { MCI brainrate temporal 30 } & -0.069 & -1.064 & 0.925 \\ 10 & \text { W } & \text { MCI complexity central 30 } & 0.094 & -0.464 & 0.652 \\ 22 & \text { W } & \text { MCI complexity frontal 30 } & 0.131 & -0.768 & 1.031 \\ 34 & \text { W } & \text { MCI complexity temporal 30 } & 0.121 & -0.652 & 0.895 \\ 6 & \text { W } & \text { SCC brainrate central 47 } & 0.537 & -0.049 & 1.124 \\ 18 & \text { W } & \text { SCC brainrate frontal } 47 & 0.548 & -0.062 & 1.159 \\ 30 & \text { W } & \text { SCC brainrate temporal } 47 & 0.559 & -0.015 & 1.133 \\ 12 & \text { W } & \text { SCC complexity central 47 } & 0.384 & 0.110 & 0.659 \\ 24 & \text { W } & \text { SCC complexity frontal 47 } & 0.403 & -0.038 & 0.845 \\ 36 & \text { W } & \text { SCC complexity temporal } 47 & 0.506 & 0.132 & 0.880\end{array}$

Wald-Type Statistic (WTS):

\begin{tabular}{|c|c|c|c|}
\hline \multicolumn{2}{|c|}{ Test statistic } & \multicolumn{2}{|l|}{ df $p$-value } \\
\hline sex & "9.973" & "1" "0.002" & \\
\hline diagnosis & $" 42.383 "$ & $" 2 " 1<0.001 "$ & \\
\hline sex:diagnosis & $" 3.777 "$ & "2" "0.151" & \\
\hline feature & $" 0.086 "$ & "1" "0.769" & \\
\hline sex: feature & "2.167" & $" 1 " 0.141 "$ & \\
\hline diagnosis: feature & $" 5.317 "$ & "2" "0.07" & \\
\hline sex:diagnosis: feature & $" 1.735 "$ & "2" "0.42" & \\
\hline region & $" 0.07 "$ & "2" "0.966" & \\
\hline sex:region & $" 0.876 "$ & "2" "0.645" & \\
\hline diagnosis:region & $" 6.121 "$ & "4" "0.19" & \\
\hline sex:diagnosis:region & $" 1.532 "$ & "4" "0.821" & \\
\hline feature:region & $" 0.652 "$ & "2" "0.722" & \\
\hline sex:feature:region & $" 0.423 "$ & "2" "0.81" & \\
\hline diagnosis: feature: region & $" 7.142 "$ & "4" "0.129" & \\
\hline sex:diagnosis: feature: region & "2.274" & "4" "0.686" & \\
\hline \multicolumn{4}{|l|}{ ANOVA-Type Statistic (ATS): } \\
\hline Test st & atistic & df2 & p-value \\
\hline $\operatorname{sex}$ & "9.973" & $" 657.416 "$ & $" 0.002 "$ \\
\hline diagnosis & $" 13.124 "$ & "1.343" "657.416" & $" \quad "<0.001 "$ \\
\hline sex:diagnosis & $" 1.904 "$ & "1.343" "657.416" & $"=0.164 "$ \\
\hline feature & $" 0.086 "$ & "Inf" & $" 0.769 "$ \\
\hline sex: feature & "2.167" & "Inf" & $" 0.141 "$ \\
\hline diagnosis: feature & $" 1.437 "$ & "1.562" "Inf" & $" 0.238 "$ \\
\hline sex:diagnosis:feature & $" 1.031 "$ & "1.562" "Inf" & $" 0.342 "$ \\
\hline region & $" 0.018 "$ & "1.611" "Inf" & $" 0.965 "$ \\
\hline sex:region & $" 0.371 "$ & "1.611" "Inf" & $" 0.644 "$ \\
\hline diagnosis:region & $" 1.091 "$ & "2.046" "Inf" & $" 0.337 "$ \\
\hline sex:diagnosis:region & $" 0.376 "$ & "2.046" "Inf" & $" 0.691 "$ \\
\hline feature:region & $" 0.126 "$ & "1.421" "Inf" & $" 0.81 "$ \\
\hline sex:feature:region & $" 0.077 "$ & "1.421" "Inf" & $" 0.864 "$ \\
\hline diagnosis: feature: region & $" 0.829 "$ & "1.624" "Inf" & $" 0.415 "$ \\
\hline sex:diagnosis: feature: region & $" 0.611 "$ & "1.624" "Inf" & $" 0.51 "$ \\
\hline
\end{tabular}

p-values resampling:

$\begin{array}{lll}\text { sex } & "<0.001 " & "<0.001 " \\ \text { diagnosis } & "<0.001 " & "<0.001 " \\ \text { sex:diagnosis } & " 0.119 " & " 0.124 "\end{array}$




$\begin{array}{lll}\text { feature } & " 0.798 " & " 0.798 " \\ \text { sex:feature } & " 0.152 " & " 0.152 " \\ \text { diagnosis:feature } & " 0.067 " & " 0.249 " \\ \text { sex:diagnosis:feature } & " 0.445 " & " 0.362 " \\ \text { region } & " 0.967 " & " 0.98 " \\ \text { sex:region } & " 0.691 " & " 0.728 " \\ \text { diagnosis:region } & " 0.182 " & " 0.338 " \\ \text { sex:diagnosis:region } & " 0.863 " & " 0.814 " \\ \text { feature:region } & " 0.814 " & " 0.926 " \\ \text { sex:feature:region } & " 0.881 " & " 0.951 " \\ \text { diagnosis:feature:region } & " 0.098 " & " 0.519 " \\ \text { sex:diagnosis:feature:region } & 0.764 " & " 0.683 "\end{array}$

We find significant effects at level $\alpha=0.01$ of the between subject factors sex and diagnosis, while none of the within subject factors or interactions become significant.

\section{Plotting}

The $\mathrm{RM}($ ) function is equipped with a plotting option, displaying the calculated means along with $(1-\alpha)$ confidence intervals based on $t$-quantiles. The plot function takes an RM object as argument. In addition, the factor of interest may be specified. If this argument is omitted in a two- or higherway layout, the user is asked to specify the factor for plotting. Furthermore, additional graphical parameters can be used to customize the plots. The optional argument legendpos specifies the position of the legend in higher-way layouts, whereas gap (default 0.1) is the distance introduced between error bars in a higher-way layout. Additionally, the parameter CI. info can be set to TRUE in order to output the means and confidence intervals for the desired interaction.

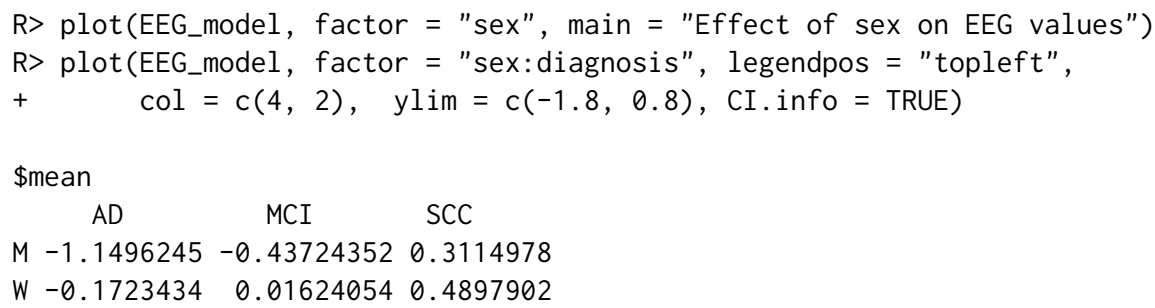

The resulting plots are displayed in Figure 1 and Figure 2, respectively.

\section{MANOVA Design}

For the analysis of multivariate data, the functions MANOVA and MANOVA. wide are implemented. The difference between the two functions is that the response must be stored in long and wide format for using MANOVA or MANOVA. wide, respectively. The structure of both functions is very similar. They both calculate the WTS for multivariate data in a design with crossed or nested factors. Additionally, the modified ANOVA-type statistic (MATS) is calculated which has the additional advantage of being applicable to designs involving singular covariance matrices and is invariant under scale transformations of the data (Friedrich and Pauly, 2018). The resampling methods provided are a parametric bootstrap approach and a wild bootstrap using Rademacher weights. Note that only balanced nested designs (i.e., the same number of factor levels $b$ for each level of the factor $A$ ) with up to three factors are implemented. Designs involving both crossed and nested factors are not implemented. Note that in nested designs, the levels of the nested factor usually have the same labels 


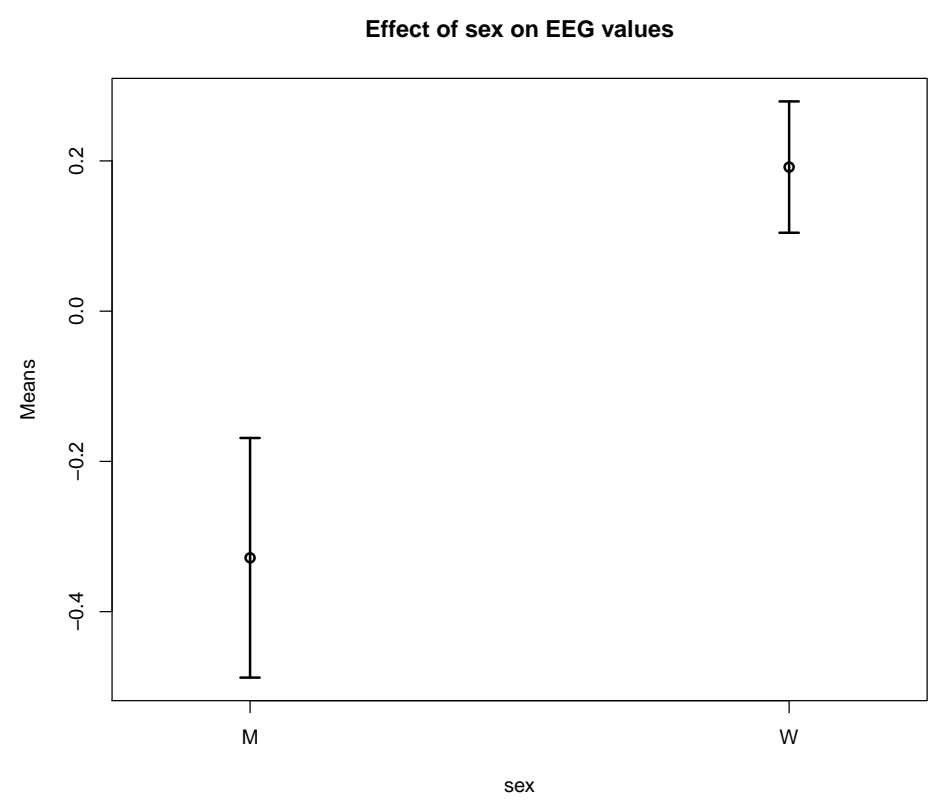

Figure 1: Plot for factor "sex" in the RM model of the EEG data example.

for all levels of the main factor, i.e., for each level $i=1, \ldots, a$ of the main factor $A$ the nested factor levels are labeled as $j=1, \ldots, b_{i}$. If the levels of the nested factor are named uniquely, this has to be specified by setting the parameter nested. levels. unique to TRUE.

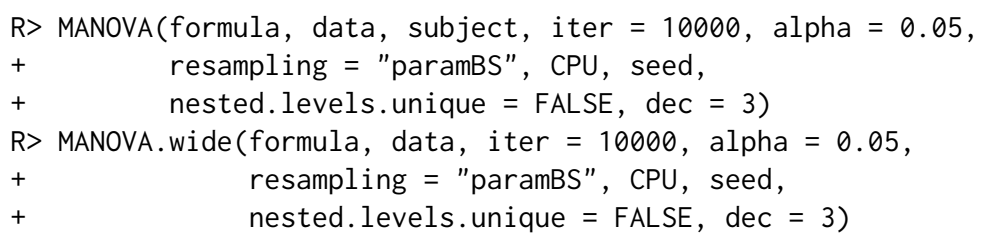

The only difference between MANOVA and MANOVA. wide in the function call except from the different shape of the formula (see examples below) is the subject variable, which needs to be specified for MANOVA only.

\section{Data Example MANOVA: Two crossed factors}

We again consider the data set EEG from the MANOVA.RM package, but now we ignore the within subject factor structure. Therefore, we are now in a multivariate setting with 6 measurements per patient and three crossed factors sex, age and diagnosis. Due to the small number of subjects in some groups we restrict our analyses to two factors at a time. The analysis of this example is shown below.

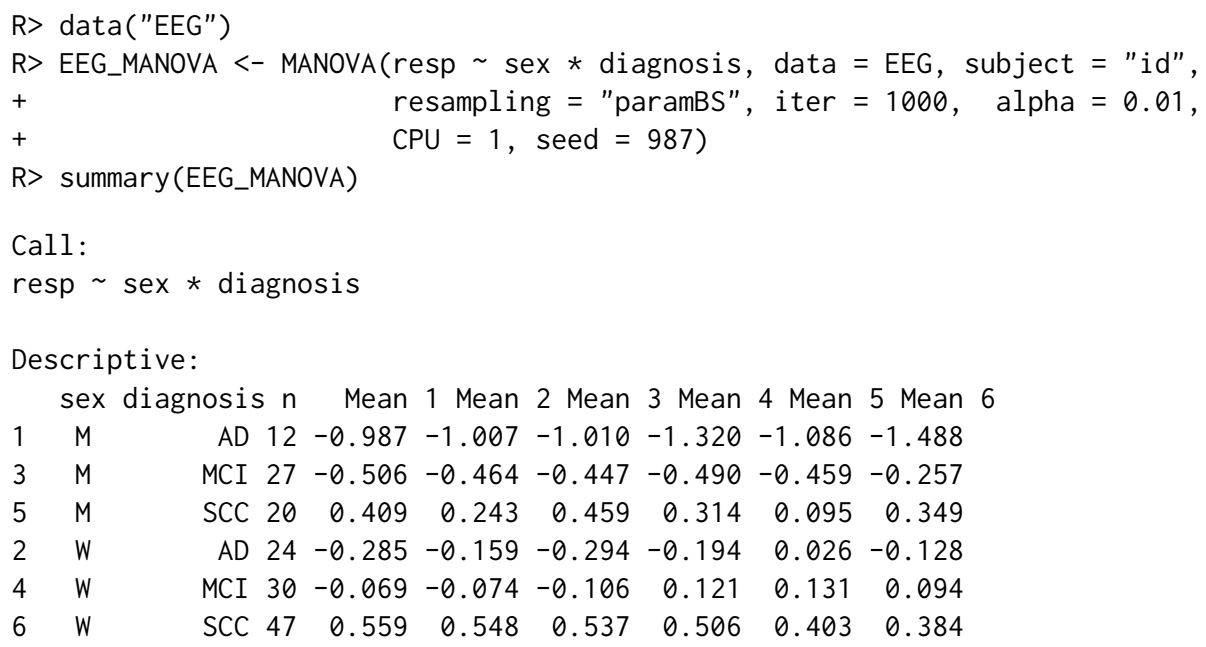



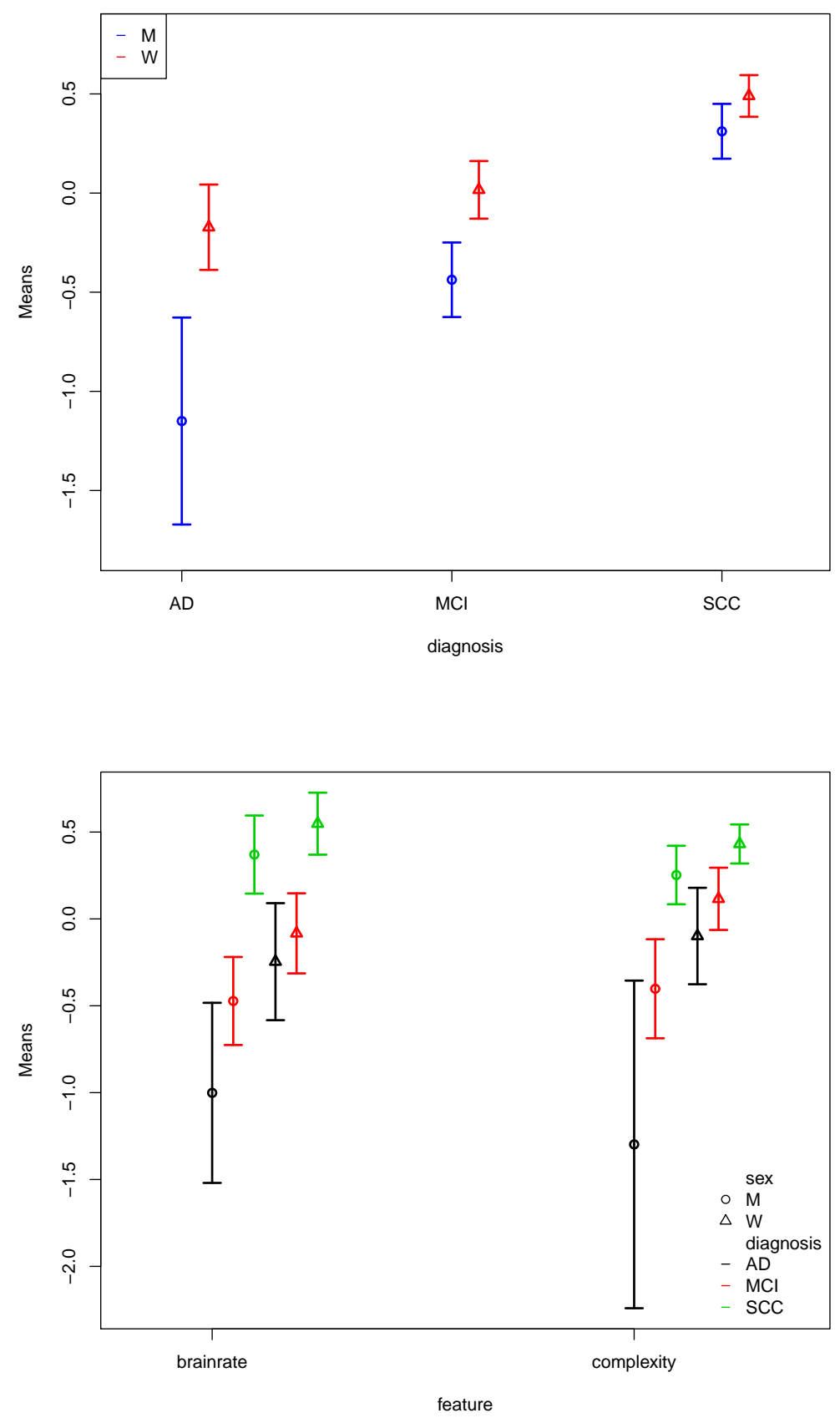

Figure 2: Plot for the interaction between "sex" and "diagnosis" (upper panel) as well as additionally taking "feature" into account (lower panel) in the RM model of the EEG data example. 


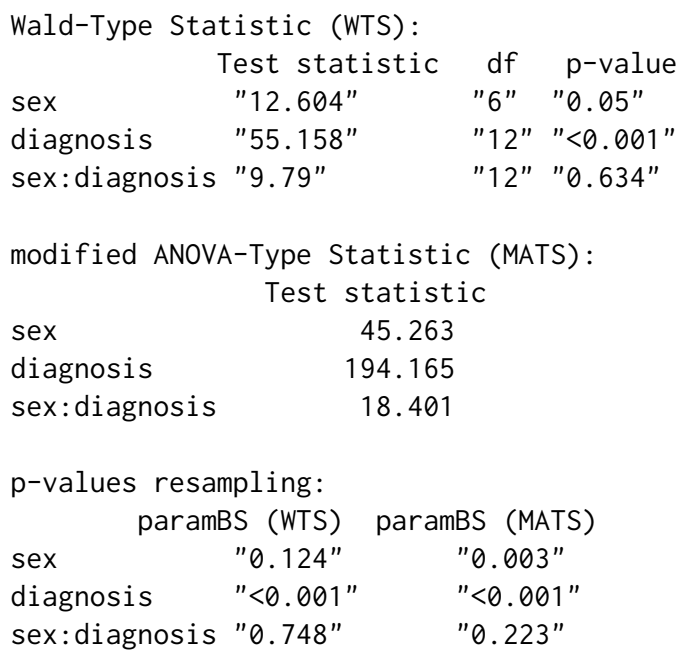

The output consists of several parts: First, some descriptive statistics of the data set are displayed, namely the sample size and mean for each factor level combination and each dimension (dimensions occur in the same order as in the original data set). In this example, Mean 1 to Mean 3 correspond to the brainrate (temporal, frontal, central) while Mean 4-6 correspond to complexity. Second, the results based on the WTS are displayed. For each factor, the test statistic, degree of freedom and $p$ value is given. For the MATS, only the value of the test statistic is given, since here inference is only based on resampling. The resampling-based $p$ values are finally displayed for both test statistics.

To demonstrate the use of the MANOVA.wide() function, we consider the same data set in wide format, which is also included in the package. In the formula argument, the user now needs to specify the variables of interest bound together via cbind. A subject variable is no longer necessary, as every row of the data set belongs to one patient in wide format data. The output is almost identically to the one obtained from MANOVA with the difference that the mean values are now labeled according to the variable names supplied in the formula argument.

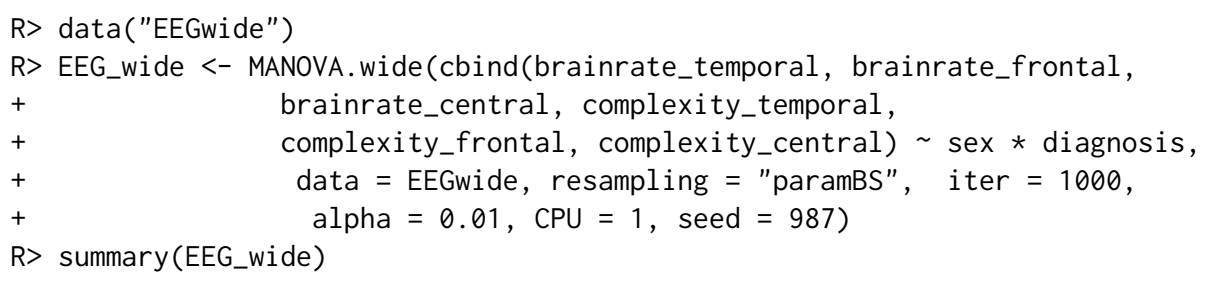

Call:

cbind(brainrate_temporal, brainrate_frontal, brainrate_central, complexity_temporal, complexity_frontal, complexity_central) sex * diagnosis

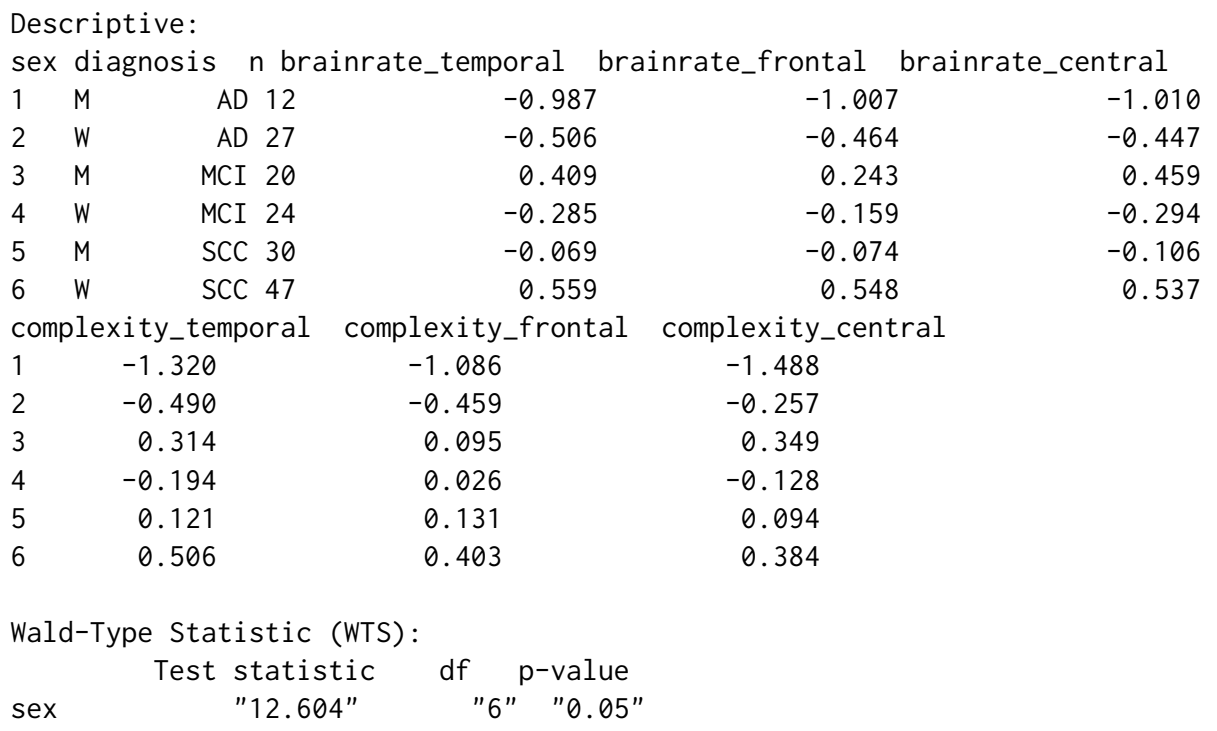




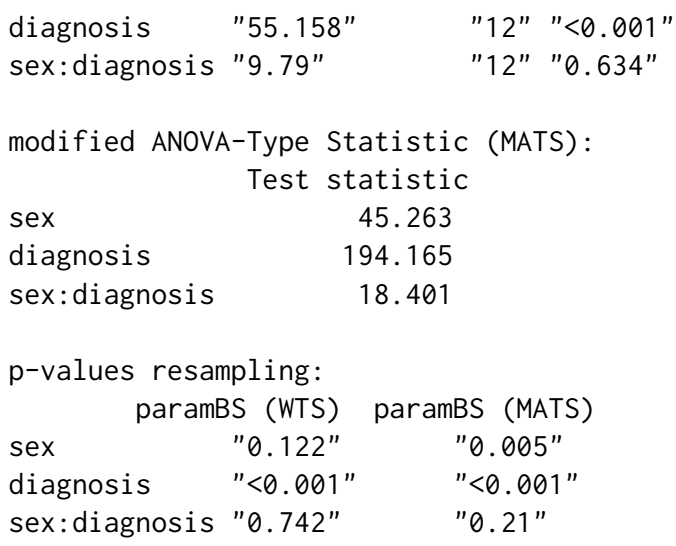

In this example, MATS detects a significant effect of sex, a finding that is not shared by the $p$ value based on the parametric bootstrap WTS.

\section{Confidence Regions}

The MANOVA functions are equipped with a function for calculating and plotting of confidence regions. Details on the methods can be found in Friedrich and Pauly (2018). We would like to point out, however, that the MATS-based confidence regions have to be interpreted differently from the more usual WTS-based ones. For the latter, the WTS is compared to a fixed critical value (in the asymptotic choice from a $\chi^{2}$-distribution) and we thus obtain geometric ellipsoids as the WTS is more or less a Mahalanobis distance in the inverse covariance matrix. The MATS statistic only involves the variances of the covariance matrix and we thus obtain a different geometric shape of the corresponding confidence region. However, here the correlation is implicitly involved in the critical value which now (different to the WTS) depends on the covariance matrix. More precisely, a confidence region for the vector of contrasts $\boldsymbol{H} \boldsymbol{\mu}$ based on the MATS is determined by the set of all $\boldsymbol{H} \boldsymbol{\mu}$ such that

$$
N(\boldsymbol{H} \overline{\boldsymbol{X}} \cdot-\boldsymbol{H} \boldsymbol{\mu})^{\top}\left(\boldsymbol{H} \widehat{\boldsymbol{D}}_{N} \boldsymbol{H}^{\top}\right)^{+}(\boldsymbol{H} \overline{\boldsymbol{X}} \cdot-\boldsymbol{H} \boldsymbol{\mu}) \leq c_{1-\alpha}^{*}
$$

where $c_{1-\alpha}^{*}$ denotes the quantile of the respective resampling distribution. A confidence ellipsoid is now obtained based on the eigenvalues $\widehat{\lambda}_{s}$ and eigenvectors $\widehat{\boldsymbol{e}}_{S}$ of $\boldsymbol{H} \widehat{\boldsymbol{D}}_{N} \boldsymbol{H}^{\top}$. The classical WTS-based confidence ellipsoids, in contrast, are based on eigenvectors and eigenvalues of $\boldsymbol{H} \widehat{\boldsymbol{\Sigma}}_{N} \boldsymbol{H}^{\top}$ instead. Confidence regions can be calculated using the conf. reg function. Note that confidence regions can only be plotted in designs with 2 dimensions.

$\mathrm{R}>$ conf.reg(object, nullhypo)

Object must be an object of class "MANOVA", i.e., created using either MANOVA or MANOVA.wide, whereas nullhypo specifies the desired null hypothesis, i.e., the contrast of interest in designs involving more than one factor. As an example, we consider the data set water from the HSAUR package (Everitt and Hothorn, 2017). The data set contains measurements of mortality and drinking water hardness for 61 cities in England and Wales. Suppose we want to analyse whether these measurements differ between northern and southern towns. Since the data set is in wide format, we need to use the MANOVA. wide function.

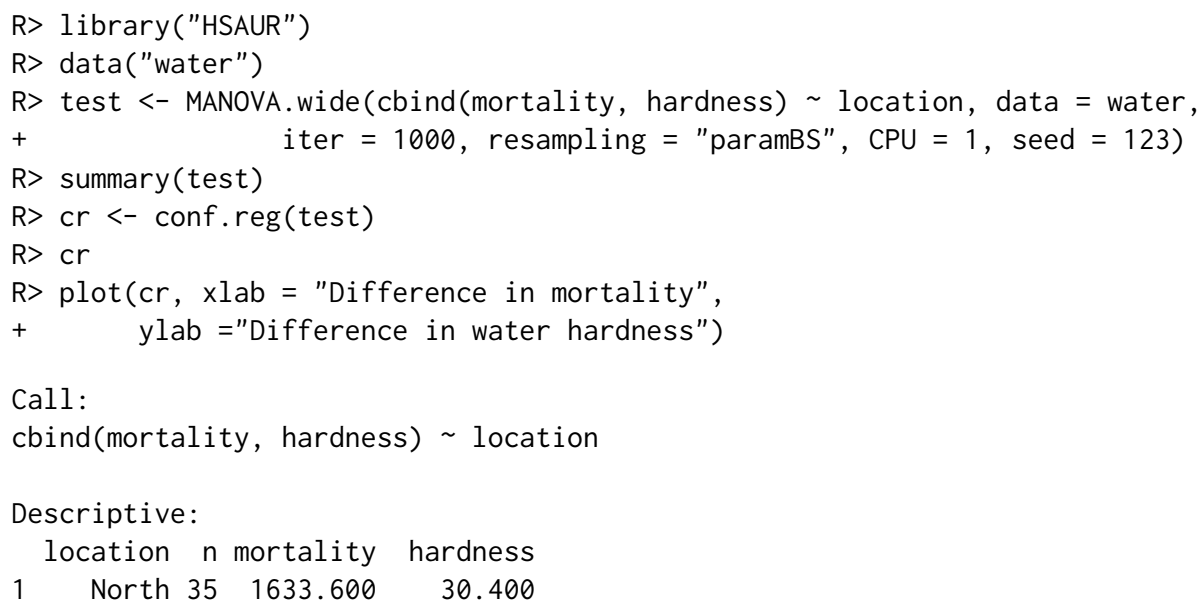


2

Wald-Type Statistic (WTS):

Test statistic df p-value

location "51.584" "2" "<0.001"

modified ANOVA-Type Statistic (MATS):

Test statistic

location $\quad 69.882$

p-values resampling:

paramBS (WTS) paramBS (MATS)

location "<0.001" $\quad<0.001 "$

We find significant differences in mortality and water hardness between northern and southern towns.

The confidence region is returned as an ellipsoid specified by its center as well as its axes, which extend Scale units into the direction of the respective eigenvector. For two-dimensional outcomes as in this example, the confidence ellipsoid can also be plotted using the ellipse package (Murdoch and Chow, 2018), see Figure 3.

Center:

$[, 1]$

$[1]$,

$[2]-$,

Scale:

[1] $10.852716 \quad 2.736354$

Eigenvectors:

$[, 1][, 2]$

$[1] \quad-,1 \quad 0$

$[2] \quad 0 \quad-$,

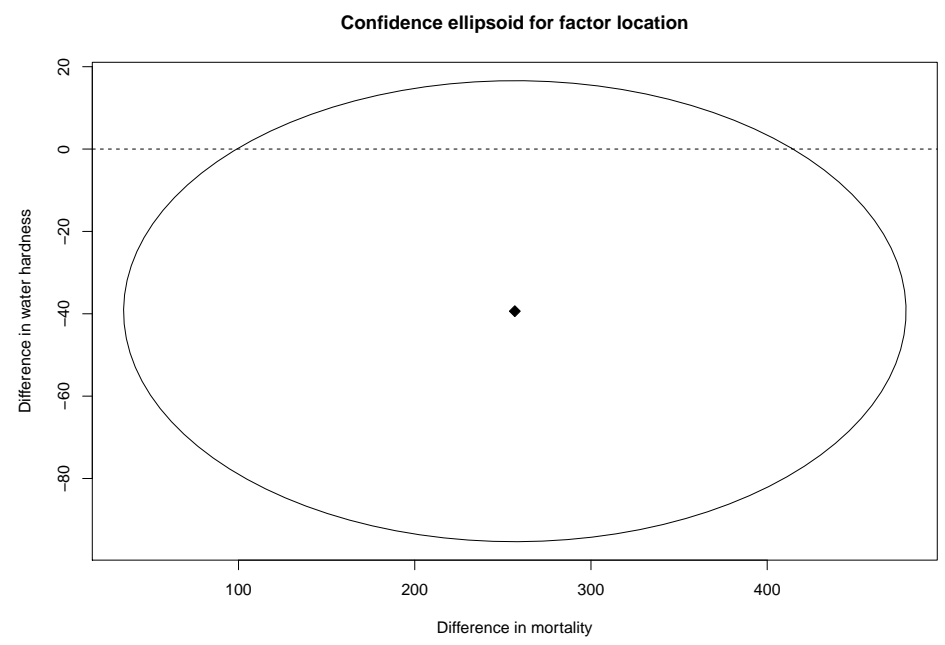

Figure 3: Plot of the confidence region for factor location in the water example from package HSAUR.

\section{Nested design}

To create a data example for a nested design, we use the curdies data set from the GFD package and extend it by introducing an artificial second outcome variable. In this data set, the levels of the nested factor (site) are named uniquely, i.e., levels 1-3 of factor site belong to "WINTER", whereas levels 4-6 belong to "SUMMER". Therefore, nested. levels. unique must be set to TRUE. The code for the analysis using both wide and long format is presented below. 


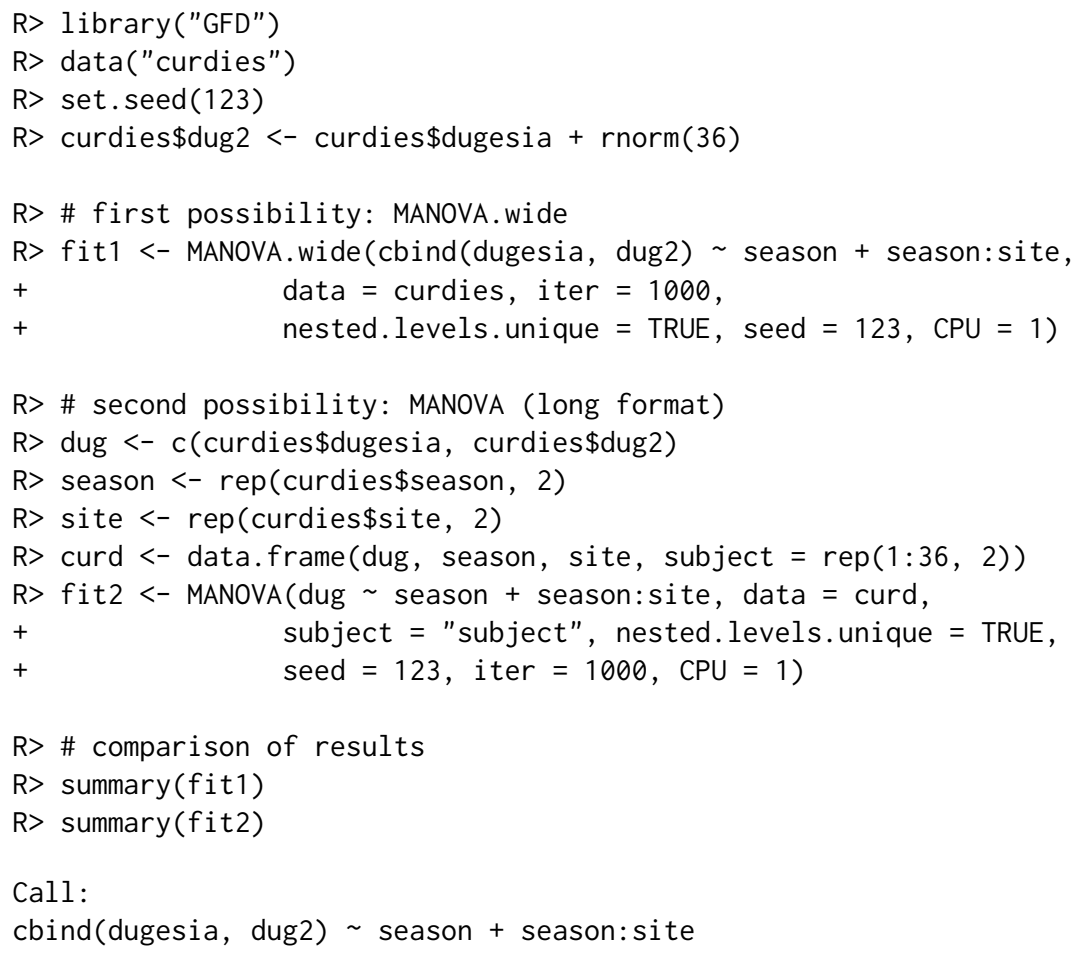

Call:

cbind(dugesia, dug2) season + season:site

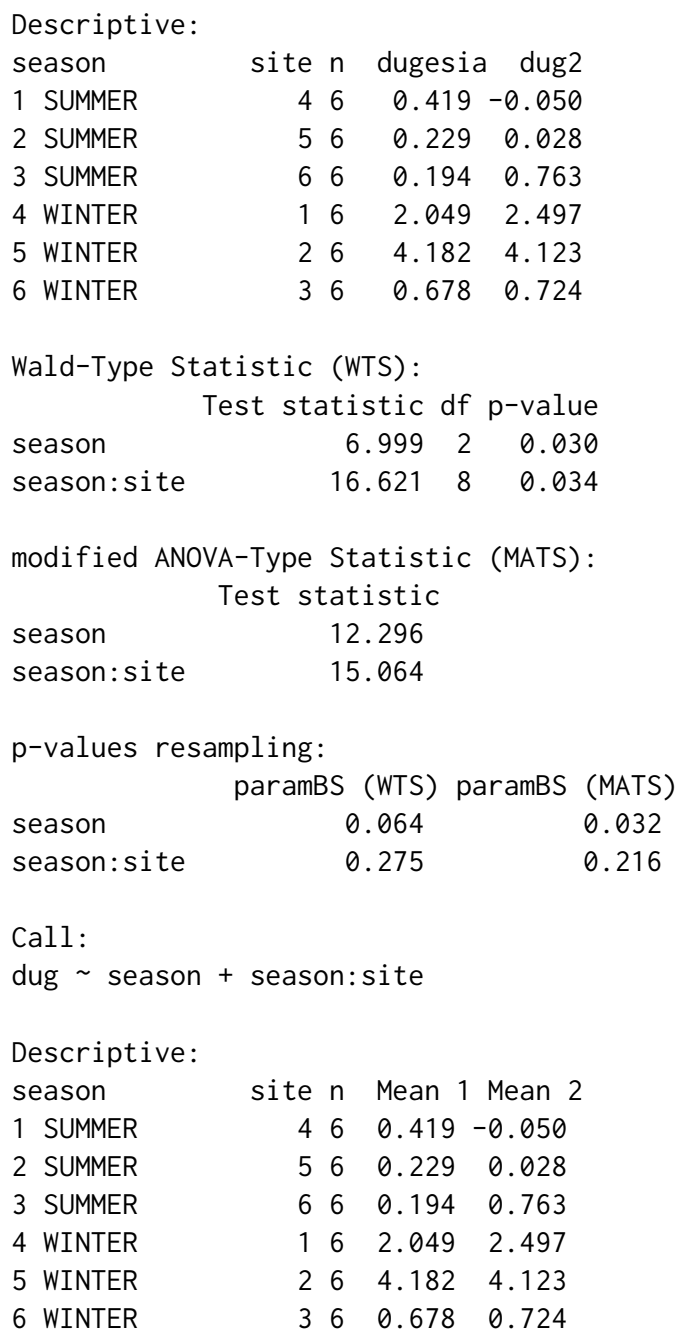




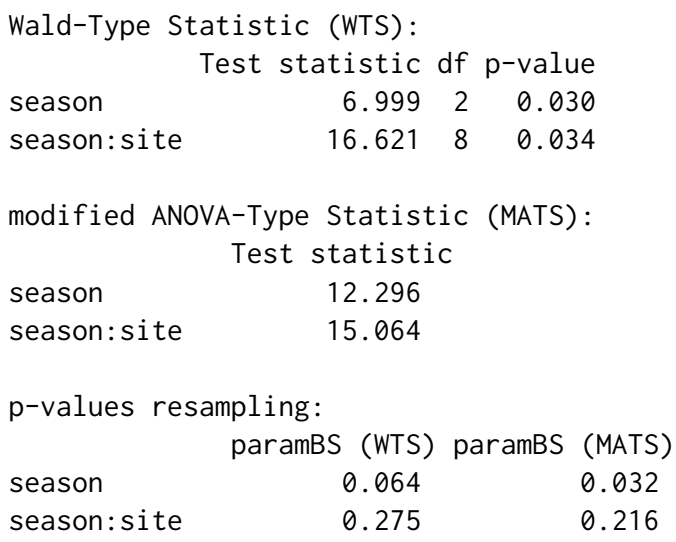

\section{Post-hoc comparisons}

In addition to global testing, the package MANOVA.RM allows for post-hoc comparisons. In particular, the following comparisons are implemented:

1. calculation of simultaneous multivariate $p$ values for contrasts of the mean vector,

2. calculation of simultaneous confidence intervals based on summary effects (i. e. averaged across all dimensions) and

3. univariate comparisons for separate endpoints.

Calculation of simultaneous confidence intervals and $p$ values for contrasts of the mean vector is based on the sum statistic, see Friedrich and Pauly (2018) for details. Confidence intervals are calculated based on summary effects, i.e., averaging over all dimensions, whereas the returned pvalues are multivariate. Note that the confidence intervals and $p$ values returned are simultaneous, i. e., they maintain the given alpha-level. Such contrasts include, e. g., Tukey's all-pairwise comparisons or Dunnett's many-to-one comparisons, see e. g. Hothorn et al. (2008a) for more examples. Confidence intervals for contrasts of the mean vector can be calculated using the function simCI, which is build on contrMat from the multcomp package (Hothorn et al., 2008b):

simCI (object, contrast, contmat, type, base)

Here, object is an object of class "MANOVA". The user can choose between pairwise or user-defined contrasts. For user-defined constrast (contrast = "user-defined"), the contrast matrix of interest must be specified in contmat. Pairwise comparisons (contrast = "pairwise") are calculated using the contrMat function of multcomp and accordingly, type and base specify the type of the pairwise comparison and the baseline group for Dunnett contrasts, see Hothorn et al. (2008b) for details on these parameters. To exemplify its application we reconsider the EEG example from above:

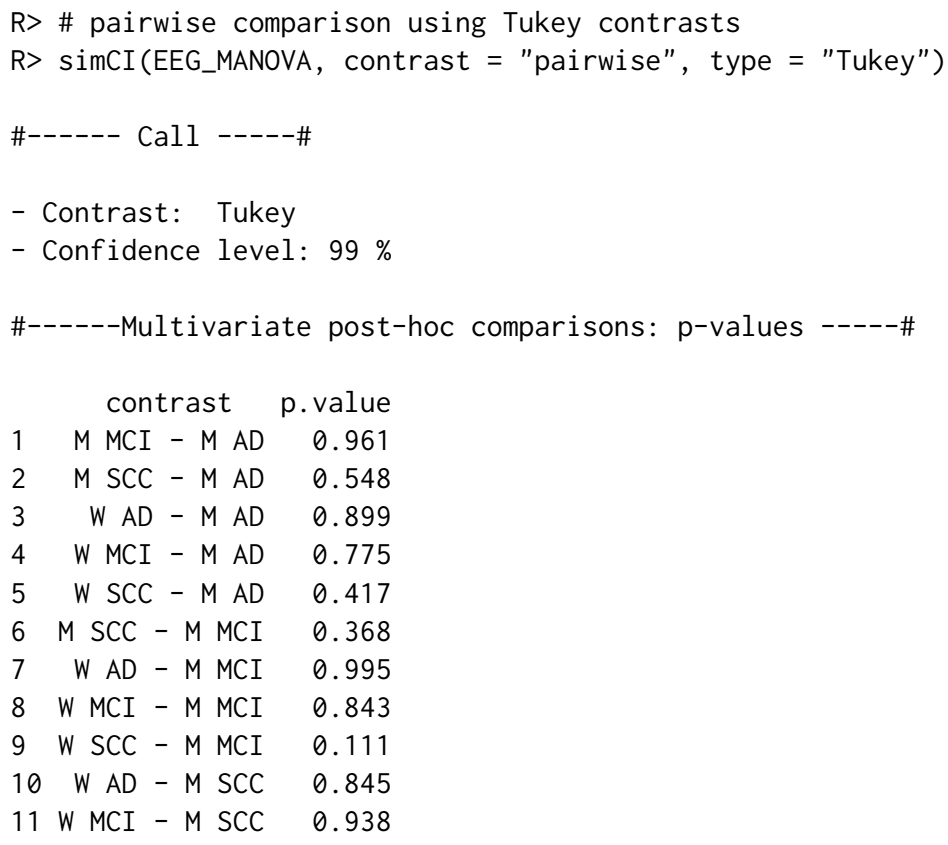




$\begin{array}{lll}12 \text { W SCC - M SCC } & 0.989 \\ 13 \text { W MCI - W AD } & 1.000 \\ 14 \text { W SCC - W AD } & 0.526 \\ 15 \text { W SCC - W MCI } & 0.563\end{array}$

\#------------Confidence intervals for summary effects--------------\#

\begin{tabular}{|c|c|c|c|}
\hline & stimate & er & \\
\hline$M C I-M A D$ & 4.275 & -16.181707 & $2^{2}$ \\
\hline$C C-M A D$ & & -1 & \\
\hline$-M A D$ & & & \\
\hline$I-M A D$ & & & \\
\hline$C-M A D$ & & & \\
\hline C & & & \\
\hline$t$ & & -8 & \\
\hline$C I-M$ & 0 & -5 & \\
\hline MCI & & -2.3 & 13 \\
\hline$-M$ & -2.903 & -12.2 & 6.4 \\
\hline MCI - M SCC & -1.772 & -9.0 & 5.5 \\
\hline$C C-M S C C$ & & -5.2 & \\
\hline$C I-W A D$ & & -8.3 & 10.6 \\
\hline$C-W$ & 3.9 & -4.8 & 12.7 \\
\hline- - W N & & $-3.71914 e$ & ובתבנ. \\
\hline
\end{tabular}

The output is two-fold: First, the multivariate $p$ values for the desired contrasts are returned. The second part of the output provides simultaneous confidence intervals for summary effects by averaging over all dimensions.

As another example using a user-defined contrast matrix, we consider the following one-way layout of the EEG data:

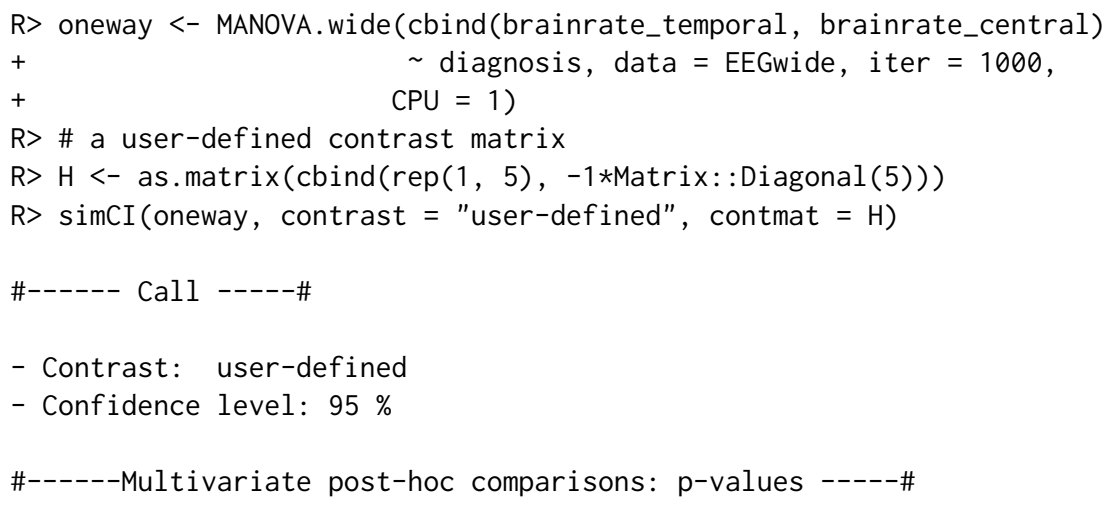

[1] $\begin{array}{lllll}1.000 & 0.673 & 0.655 & 0.008 & 0.008\end{array}$

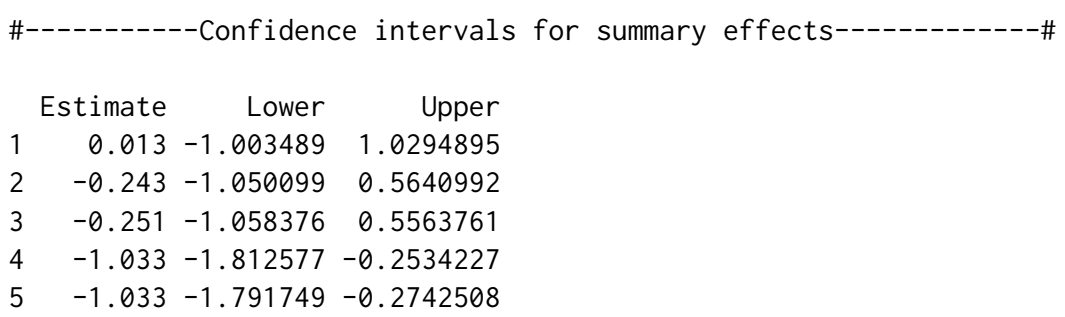

Note that interpretation of the results depends on the user-defined contrast matrix.

If the global null hypothesis, e. g.

$$
H_{0}(\boldsymbol{A}):\left\{\left(\boldsymbol{P}_{a} \otimes \boldsymbol{I}_{d}\right) \boldsymbol{\mu}=\mathbf{0}\right\}=\left\{\boldsymbol{\mu}_{1}=\cdots=\boldsymbol{\mu}_{a}\right\}
$$

has been rejected, it is usually of interest to further investigate which univariate endpoints caused the rejection. A straight-forward way to do this is to calculate the univariate $p$ values and adjust them for multiple testing, e. g., using Bonferroni correction. Consider the one-way layout above: Since the global null hypothesis can be rejected, we now wish to analyze which of the two univariate endpoints caused this rejection. 


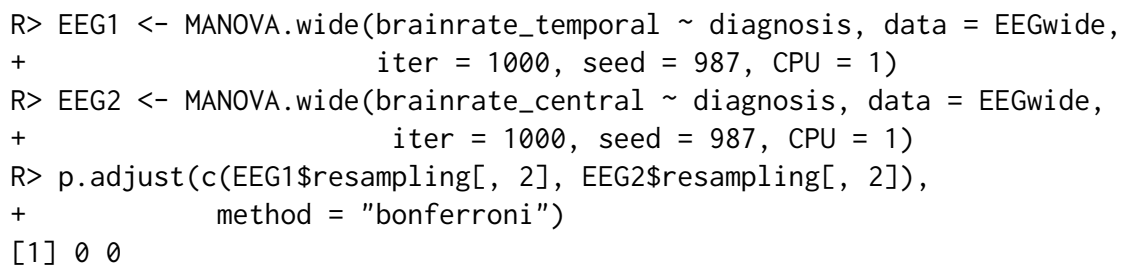

Thus, in this example both endpoints showed significant effects.

Note that it is often possible to conduct post-hoc comparisons according to the closure principle, thus avoiding the need to correct for multiple comparisons. Implementation of these methods for both MANOVA and RM designs is part of future research.

\section{Graphical user interface}

The GUI is started in R with the command GUI.RM(), GUI. MANOVA() and GUI.MANOVAwide() for repeated measures designs and multivariate data in long or wide format, respectively. Note that the GUI depends on RGtk $\mathbf{2}$ and will only work if RGtk $\mathbf{2}$ is installed. The user can specify the data location (either directly or via the "load data" button) and the formula as well as the number of iterations, the significance level $\alpha$, the number of within subject factors (for repeated measures designs) and the name of the subject variable, see Figure 4. Furthermore, the user has the choice between the three resampling approaches "Perm" (only for RM designs), "paramBS" and "WildBS" denoting the permutation procedure, the parametric bootstrap and the wild bootstrap, respectively. Additionally, one can specify whether or not headers are included in the data file, and which separator and character symbols are used for decimals in the data file. The GUI for repeated measures also provides a plotting option, which generates a new window for specifying the factors to be plotted (in higher-way layouts) along with a few plotting parameters, see Figure 5.

R> library ("MANOVA.RM")

$\mathrm{R}>\mathrm{GUI} . \mathrm{RM}()$
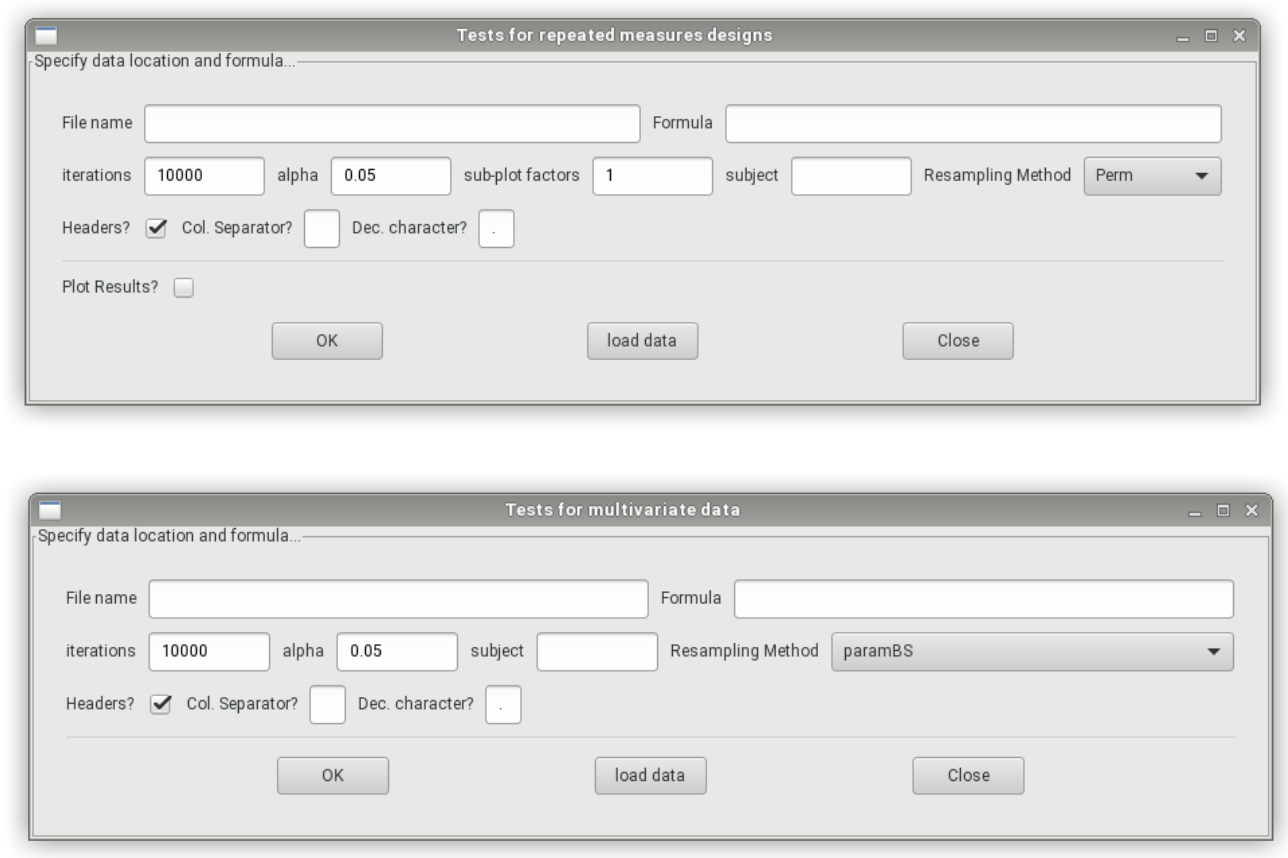

Figure 4: The GUI for tests in repeated measures designs (upper panel) and multivariate data (lower panel): The user can specify the data location and the formula as well as the resampling approach. 

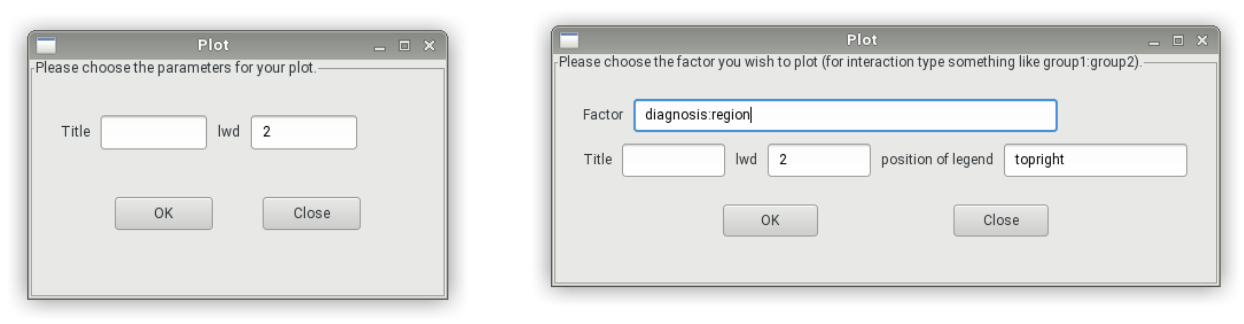

Figure 5: Graphical user interfaces for plotting: The left GUI is for the one-way layout (no choice of factors possible), the right one is for a two-way layout with an example for plotting interactions.

\section{Discussion and Outlook}

We have explicitly described the usage of the R package MANOVA.RM for analyzing various nonparametric multivariate MANOVA and RM designs making use of novel bootstrap- and permutationapproaches. Moreover, the corresponding models and inference procedures that have been derived and theoretically analyzed in previous papers are explained as well. In particular, three different test statistics of Wald-, ANOVA- and modified ANOVA-type are implemented together with appropriate critical values derived from asymptotic considerations, approximations or novel resampling approaches. Here, the latter is recommended in case of small to moderate sample sizes. All methods can be applied without assuming usual presumptions such as multivariate normality or specific covariance structures. Moreover, all procedures are particularly constructed to tackle covariance matrix heterogeneity across groups or even covariance singularity (in case of the MATS). In this way MANOVA.RM provides a flexible tool box for inferring hypotheses about (i) main and interaction effects in general factorial MANOVA and (ii) between and within subject effects in RM designs with possibly complex factorial structures on both, between and within subject factors.

In addition, we have placed a graphical user interface (GUI) at the users disposal to allow for a simple and intuitive use. It is planned to update the package on a regular basis; respecting the development of new procedures for general RM and MANOVA designs. For example, our working group is currently investigating the implementation of covariates in the above model in theoretical research and the resulting procedure may be incorporated in the future. Other topics include the possible implementation/improvement of subsequent multiple comparisons by the closure principle, as in Burchett et al. (2017), for both MANOVA and RM designs.

\section{Acknowledgments}

The work of Sarah Friedrich and Markus Pauly was supported by the German Research Foundation project DFG-PA 2409/3-1.

\section{Bibliography}

M. J. Anderson. A new method for non-parametric multivariate analysis of variance. Austral Ecology, 26(1):32-46, 2001. [p380]

A. C. Bathke, S. Friedrich, F. Konietschke, M. Pauly, W. Staffen, N. Strobl, and Y. Höller. Testing Mean Differences Among Groups: Multivariate and Repeated Measures Analysis with Minimal Assumptions. Multivariate Behavioral Research, 2018. URL https://doi .org/10.1080/00273171. 2018. 1446320. [p381, 382, 386]

E. Brunner. Asymptotic and approximate analysis of repeated measures designs under heteroscedasticity. Mathematical Statistics with Applications in Biometry, 2001. [p381, 382, 383, 386]

E. Brunner, H. Dette, and A. Munk. Box-type approximations in nonparametric factorial designs. Journal of the American Statistical Association, 92(440):1494-1502, 1997. [p383]

E. Brunner, S. Domhof, and F. Langer. Nonparametric Analysis of Longitudinal Data in Factorial Experiments. John Wiley \& Sons, New York, USA, 2002. [p383]

W. W. Burchett, A. R. Ellis, S. W. Harrar, and A. C. Bathke. Nonparametric inference for multivariate data: The R package npmv. Journal of Statistical Software, 76(4):1-18, 2017. URL https: //doi .org/ 10.18637/jss.v076.i04. [p380, 398] 
W. F. Christensen and A. C. Rencher. A Comparison of Type I Error Rates and Power Levels for Seven Solutions to the Multivariate Behrens-Fisher Problem. Communications in Statistics - Simulation and Computation, 26(4):1251-1273, 1997. [p383]

E. Chung and J. P. Romano. Exact and asymptotically robust permutation tests. The Annals of Statistics, 41(2):484-507, 2013. [p381]

G. Cornu, F. Mortier, C. Trottier, and X. Bry. SCGLR: Supervised Component Generalized Linear Regression, 2018. URL https://CRAN. R-project.org/package=SCGLR. R package version 3.0. [p381]

C. S. Davis. Statistical Methods for the Analysis of Repeated Measurements. Springer-Verlag, 2002. [p380]

B. S. Everitt and T. Hothorn. HSAUR: A Handbook of Statistical Analyses Using R (1st Edition), 2017. URL https://CRAN.R-project.org/package=HSAUR. R package version 1.3-9. [p392]

L. Finos, with contributions by Florian Klinglmueller, D. Basso, A. Solari, L. Benetazzo, J. Goeman, and M. Rinaldo. Flip: Multivariate Permutation Tests, 2018. URL https://CRAN. R-project.org/package= flip. R package version 2.5.0. [p381]

J. Fox and S. Weisberg. An R Companion to Applied Regression. Sage, Thousand Oaks CA, 2nd edition, 2011. URL http://socserv.socsci.mcmaster.ca/jfox/Books/Companion. [p381]

S. Friedrich and M. Pauly. MATS: Inference for potentially singular and heteroscedastic MANOVA. Journal of Multivariate Analysis, 165:166-179, 2018. [p381, 383, 388, 392, 395]

S. Friedrich, E. Brunner, and M. Pauly. Permuting longitudinal data in spite of the dependencies. Journal of Multivariate Analysis, 153:255-265, 2017a. [p381, 382, 385, 386]

S. Friedrich, F. Konietschke, and M. Pauly. Gfd: An r package for the analysis of general factorial designs. Journal of Statistical Software, Code Snippets, 79(1):1-18, 2017b. URL https://doi . org/10. $18637 /$ jss.v079.c01. [p381]

S. Friedrich, F. Konietschke, and M. Pauly. MANOVA.RM: Resampling-Based Analysis of Multivariate Data and Repeated Measures Designs, 2019. URL http://gi thub.com/smn74/MANOVA. RM. R package version 0.4.1. [p381]

A. K. Gupta, S. W. Harrar, and Y. Fujikoshi. MANOVA for large hypothesis degrees of freedom under non-normality. Test, 17(1):120-137, 2008. [p381]

S. W. Harrar and A. C. Bathke. A modified two-factor multivariate analysis of variance: Asymptotics and small sample approximations. Annals of the Institute of Statistical Mathematics, 64(1):135-165, 2012. [p381]

T. Hothorn, F. Bretz, and P. Westfall. Simultaneous inference in general parametric models. Biometrical Journal, 50(3):346-363, 2008a. [p395]

T. Hothorn, F. Bretz, and P. Westfall. Simultaneous inference in general parametric models. Biometrical Journal, 50(3):346-363, 2008b. [p395]

Y. Huang, H. Xu, V. Calian, and J. C. Hsu. To permute or not to permute. Bioinformatics, 22(18): 2244-2248, 2006. [p381]

R. A. Johnson and D. W. Wichern. Applied Multivariate Statistical Analysis. Prentice Hall, 2007. [p380]

F. Konietschke, A. C. Bathke, S. W. Harrar, and M. Pauly. Parametric and nonparametric bootstrap methods for general MANOVA. Journal of Multivariate Analysis, 140:291-301, 2015. [p381, 382, 383, 386]

K. Krishnamoorthy and J. Yu. Modified Nel and Van Der Merwe Test for the Multivariate BehrensFisher Problem. Statistics \& Probability Letters, 66(2):161-169, 2004. [p383]

M. Lawrence and D. Temple Lang. RGtk2: A graphical user interface toolkit for R. Journal of Statistical Software, 37(8):1-52, 2010. URL http://www. jstatsoft.org/v37/i08/. [p384]

J. Lemon. Plotrix: a package in the red light district of r. R-News, 6(4):8-12, 2006. [p385]

P. Livacic-Rojas, G. Vallejo, and P. Fernandez. Analysis of type i error rates of univariate and multivariate procedures in repeated measures designs. Communications in Statistics - Simulation and Computation, 39(3):624-640, 2010. [p381] 
P. Livacic-Rojas, G. Vallejo, P. Fernández, and E. Tuero-Herrero. Power of modified brown-forsythe and mixed-model approaches in split-plot designs. Methodology, 13:9-22, 2017. [p381]

L. M. Lix and H. Keselman. Multivariate tests of means in independent groups designs: Effects of covariance heterogeneity and nonnormality. Evaluation $\mathcal{E}$ the Health Professions, 27(1):45-69, 2004. [p381]

L. M. Lix and A. M. Lloyd. A comparison of procedures for the analysis of multivariate repeated measurements. Journal of Modern Applied Statistical Methods, 6(2):380-398, 2007. [p381]

M. McFarquhar, S. McKie, R. Emsley, J. Suckling, R. Elliott, and S. Williams. Multivariate and repeated measures (mrm): A new toolbox for dependent and multimodal group-level neuroimaging data. NeuroImage, 132:373-389, 2016. [p381]

D. Murdoch and E. D. Chow. Ellipse: Functions for Drawing Ellipses and Ellipse-Like Confidence Regions, 2018. URL https://CRAN.R-project.org/package=ellipse. R package version 0.4.1. [p393]

D. Nel and C. Van der Merwe. A Solution to the Multivariate Behrens-Fisher Problem. Communications in Statistics-Theory and Methods, 15(12):3719-3735, 1986. [p383]

K. Noguchi, Y. R. Gel, E. Brunner, and F. Konietschke. nparld: An r software package for the nonparametric analysis of longitudinal data in factorial experiments. Journal of Statistical Software, 50(12):1-23, 2012. URL http://www. jstatsoft.org/v50/i12/. [p381]

G. Vallejo, A. Fidalgo, and P. Fernandez. Effects of covariance heterogeneity on three procedures for analyzing multivariate repeated measures designs. Multivariate Behavioral Research, 36(1):01-27, 2001. [p381]

G. Vallejo Seco, J. A. Gras, and M. Ato García. Comparative robustness of recent methods for analyzing multivariate repeated measures designs. Educational and Psychological Measurement, 67(3):410-432, 2007. [p381]

S. Xiao and J.-T. Zhang. Modified Tests for Heteroscedastic Two-Way MANOVA. Journal of Advanced Statistics, 1(1):1-16, 2016. [p381]

H. Yanagihara and K.-H. Yuan. Three Approximate Solutions to the Multivariate Behrens-Fisher Problem. Communications in Statistics - Simulation and Computation, 34(4):975-988, 2005. [p383]

Y. Yao. An Approximate Degrees of Freedom Solution to the Multivariate Behrens-Fisher Problem. Biometrika, 52(1/2):139-147, 1965. [p383]

J.-T. Zhang. Two-way MANOVA with unequal cell sizes and unequal cell covariance matrices. Technometrics, 53(4):426-439, 2011. [p381]

Øyvind Langsrud and B.-H. Mevik. Ffmanova: Fifty-Fifty MANOVA, 2019. URL https://CRAN.Rproject. org/package=ffmanova. $R$ package version 1.1.0. [p381]

Sarah Friedrich

Department of Medical Statistics, University Medical Center Göttingen

Humboldtallee 32, 37073 Göttingen

Germany

sarah. friedrich@med.uni-goettingen. de

Frank Konietschke

Charité - Universitätsmedizin Berlin

Corporate Member of Freie Universität Berlin

Humboldt-Universität zu Berlin, and

Berlin Institute of Health, Institute of Biometry and Clinical Epidemiology

Charitéplatz 1, 10117 Berlin

Germany

frank. konietschke@charite. de

Markus Pauly

Fakultät Statistik, Technische Universität Dortmund

44221 Dortmund

Germany

markus.pauly@tu-dortmund. de 